\title{
Italo-Mycenaean and other Aegean-influenced pottery in Late Bronze Age Italy: the case for regional production
}

\author{
Richard E. Jones ${ }^{1}$ (D) $\cdot$ Sara T. Levi ${ }^{2}$ (D) Marco Bettelli $^{3}$ (D) $\cdot$ Valentina Cannavò $^{4}$ (D)
}

Received: 15 July 2020 / Accepted: 24 November 2020 / Published online: 15 January 2021

(C) The Author(s) 2021

\begin{abstract}
Decorated Italo-Mycenaean (IM) pottery, a high-status class found and made over three centuries from the Italian Late Middle Bronze Age onwards, was the subject of a large archaeological and archaeometric enquiry published by the present authors in 2014. The present paper focuses on identifying IM's centres of production. The results of chemical analysis of IM using mainly ICP-ES make a strong case for regional production, irrespective of findspots in several parts of Italy. This accords well with the relative stylistic individuality of IM observed among the finds of IM across many parts of Italy, suggesting that IM is a powerful archaeological indicator of the way local communities were constructing and negotiating their identities at this crucial time of social and economic change at the end of the Bronze Age. A picture of more dispersed intra-regional production emerges from the combined chemical and petrographic analysis of two other pottery classes displaying Aegean influence: wheel-made Grey ware and decorated Final Bronze Age/Early Iron Age (FBA/EIA) pottery from sites in present-day Apulia and from Broglio di Trebisacce in Calabria. Potters manufacturing the former applied their knowledge of the wheel and kiln firing to handmade impasto shapes which were largely shared by local communities within a region. The results obtained for the latter reflect demands of the new elites of the emerging FBA/EIA in southern Italy to create symbols expressing a new cultural identity: this pottery's style, especially of Protogeometric, was uniform but its production was localised.
\end{abstract}

Keywords Italo-Mycenaean pottery $\cdot$ Grey ware $\cdot$ Southern Italy $\cdot$ ICP-ES $\cdot$ Petrographic analysis $\cdot$ Provenance

\section{Introduction}

Italo-Mycenaean (IM) pottery is the name given to the class of decorated Mycenaean pottery found and made in Italy. This pottery of Aegean type is distinguished from the contemporary pottery found in Italy but which was imported from the Aegean, that is, from the Mycenaean Mainland, Crete and the Islands. Whereas the presence of imports has long been

Richard E. Jones

Richard.jones@glasgow.ac.uk

Archaeology, University of Glasgow, G12 8QQ, Glasgow, UK

2 Department of Classical and Oriental Studies, Hunter College, City University of New York, 695 Park Ave, New York, NY 10065, USA

3 CNR-Istituto di Scienze del Patrimonio Culturale, Area della Ricerca di Roma 1, Strada della neve - via Salaria Km 29,300, 00010 Montelibretti, Italy

4 Dip.di Scienze Chimiche e Geologiche, Universita' di Modena e ReggioEmilia, Via Campi 103, 41125 Modena, Italy recognised in Italy, it is only more recently that as a result of systematic and interdisciplinary study of the pottery finds bearing the stylistic and technical hallmarks associated with Mycenaean pottery that the class of IM pottery has come to be fully understood and interpreted as a specific and flourishing ware made in Italy. More specifically, Lucia Vagnetti's observations on the Mycenaean-type pottery recovered from one of the sites considered in this paper, Broglio di Trebisacce in Calabria (Vagnetti 1984, 169-196), that some of their fabrics were different from those canonically associated with the Aegean that stimulated the integration of an archaeometric component to the study.

Whether imported or locally made, this pottery stands out macroscopically, being wheel-made, decorated with paint and kiln fired in contrast to the handmade indigenous pottery. Joining the decorated IM are other specialised wares whose production in Italy was also Aegean-influenced: dolia, Grey (GR), South Italian Protogeometric (PG) and Geometric (GE), the latter dating to the Iron Age.

The salient features of the phenomenon of Aegean influence in Italy during the second half of the second millennium 
$\mathrm{BC}$, which have been explored by the present authors (Jones et al. 2014), are as follows: Aegean (and Cypriot)-type pottery has been recovered at more than a hundred sites throughout many parts of Italy, mainly in the south, as well as the associated islands (Fig. 1). IM first appears in late MBA 3 in the Italian chronology (LH IIIA Aegean chronology (c. 1420$1330 \mathrm{BC})$ ), peaking in frequency in the Recent Bronze AgeEarly Final Bronze Age (LH IIIB-C (c. 1330-1100 BC)), as shown in Fig. 2 together with the comparative figures for imported Mycenaean/Aegean.

The shapes of IM follow closely those of the repertoire of decorated fine wares of the Late Bronze Age in the Aegean that are associated principally with drinking, storage/pouring and pouring (bowls, cups, kylikes, basins, craters, jars, alabastra, stirrup jars) with a very small proportion connected with storage/transport (large jars and amphorae) (Jones et al. 2014, Figs. 6.12-15) (Fig. 3). Within this general framework, there are many local innovative solutions in terms of pottery shapes, presenting major differences from the standard Aegean types (Jones et al. 2014, 426-434, 442-444, Figs. 6.12-15). Decorative motifs on IM are also based on the Aegean models but with significant departure from them as discussed below (Jones et al. 2014, 435-437, 442-444, Figs. 6.16-20) (here Fig. 4). The manner in which the three
Fig. 1 Map of Italy showing by number the locations of Aegeantype pottery (Jones et al. 2014, Fig. 2.1)

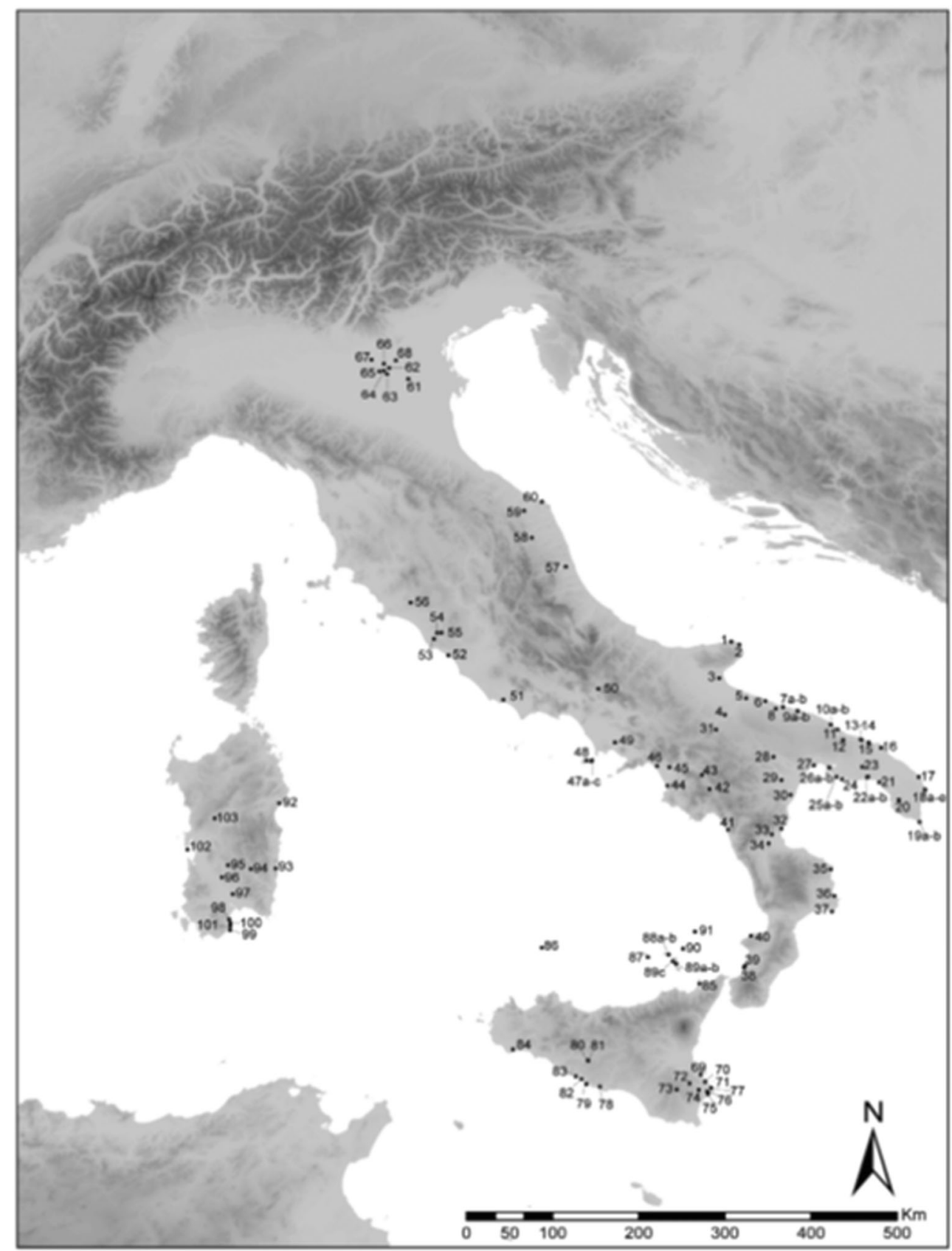


Fig. 2 Number of examples of IM and Mycenaean pottery according to the Aegean Late Helladic (I-II to IIIC) time period (Jones et al. 2014, Fig. 6.1a)

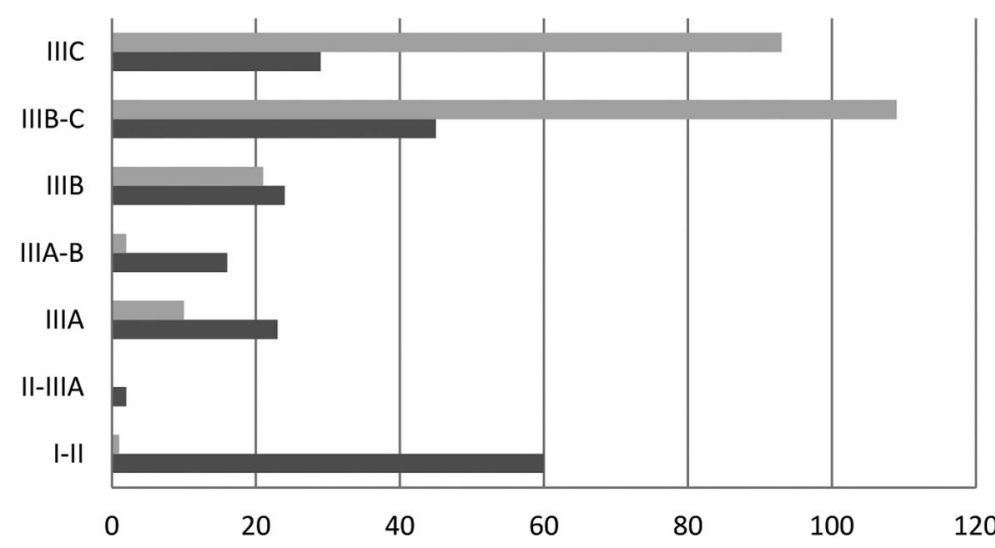

- Italo-Mycenaean

aycenaean functions - drinking, storage/pouring and pouring-continue in much the same proportions throughout LH IIIB to IIIC suggests the existence of a strong, definable tradition of specific standardised shapes inspired by Aegean models but with several local characteristics; this feature is well represented in the Plain of Sybaris (Jones et al. 2014, 415-416).

In view of the long duration of this phenomenon, almost five centuries, its chronological dimension must be introduced. The local production of Mycenaean pottery starts quite early, apparently from LH IIIA, and develops considerably in LH IIIB and IIIC both in peninsular Italy, including the Po Valley, and in Sardinia (Jones et al. 2014, 407-408; Bettelli and Levi in press). The incidence of IM productions increases progressively by some $20 \%$ from LH IIIA to LH IIIC when it becomes the essentially prevalent situation (Jones et al. 2014, Fig. 6.1b). This gradual growth seems to be linked more to an increasing demand for this type of prestige item by local communities, rather than as a consequence of the crisis of the palatial economies at the end of LH IIIB (Jones et al. 2014,
445-460). However, this general trend does not always reflect specific situations, such as, for example, those in the Plain of Sybaris and Rocavecchia or Scoglio del Tonno in Apulia. In the first case, there are very few imports and local production is in a constant majority, while at Rocavecchia and Scoglio del Tonno, imports always maintain a considerable presence, as also occurs at other sites, such as Antigori in Sardinia (Jones et al. 2014, 411-413). This phenomenon is certainly linked to the role played by the different settlements in the field of maritime-based networks. In southern Italy, wheel-thrown (and/or wheel-formed) pottery continues with no discernible gap into the Early Iron Age (EIA), with South Italian Protogeometric (PG) and Geometric (GE) wares. The maintenance of local productive networks of specialised potters using the technological package of Aegean legacy is probably to be linked to the local historical scenario. Differently from other Italian regions, south-eastern Italy, it is characterised by long-lasting settlement continuity, probably associated with a greater

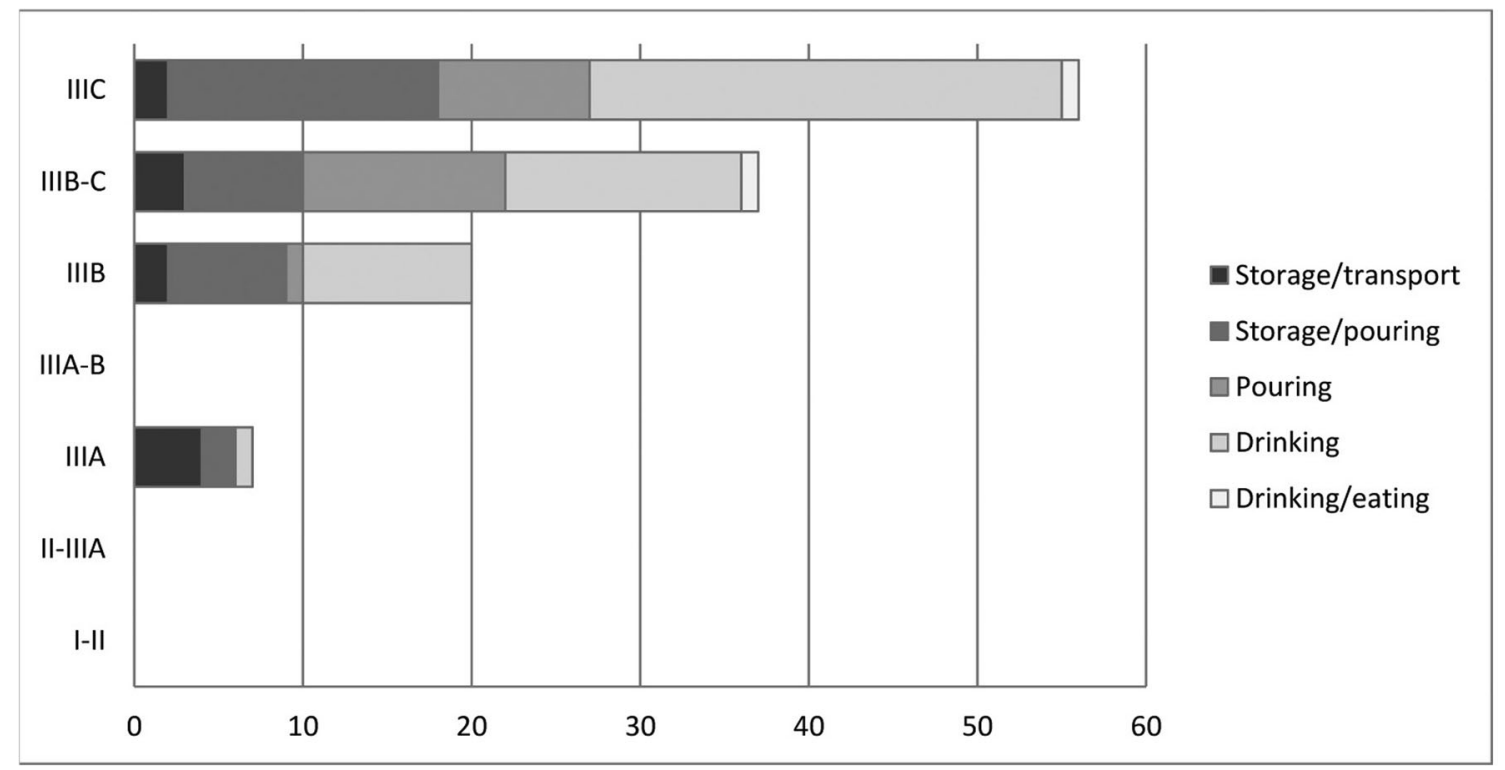

Fig. 3 IM pottery classified according to function and date (Jones et al. 2014, Fig. 6.5b) 
Fig. 4 A IM pottery from (a) Coppa Nevigata (Furumark motif (FM) 46:59), (b) Rocavecchia (FM 44:2,8), (c) Scoglio del Tonno (FM 17:23,28; 18: 27, 28), (d) Termitito (FM 75:26), (e) Broglio di Trebisacce (FM 67:6), (f) Broglio di Trebisacce Minoan flower motif, (g) Torre Mordillo (FM 70:7), (h) Pontecagnano (FM 75: 5, 18), (i) Tolentino (FM 73 row) and (j) Frattesina (FM 61). From Jones et al. 2014,

Plates 6.16-6.20 e, f, B (a) and (b) Grey ware from 'Casa Centrale' at Broglio di Trebisacce (Bettelli 2002, 233-249, Figs. 104, 33 and 103, 26. Scale 1:3). (c) and (d) decorated Protogeometric ware from Broglio di Trebisacce (Buffa 1994, 455-569, tav. 117, 14 (scale 1:3) and tav. 119, 6 (scale 1:4))
A
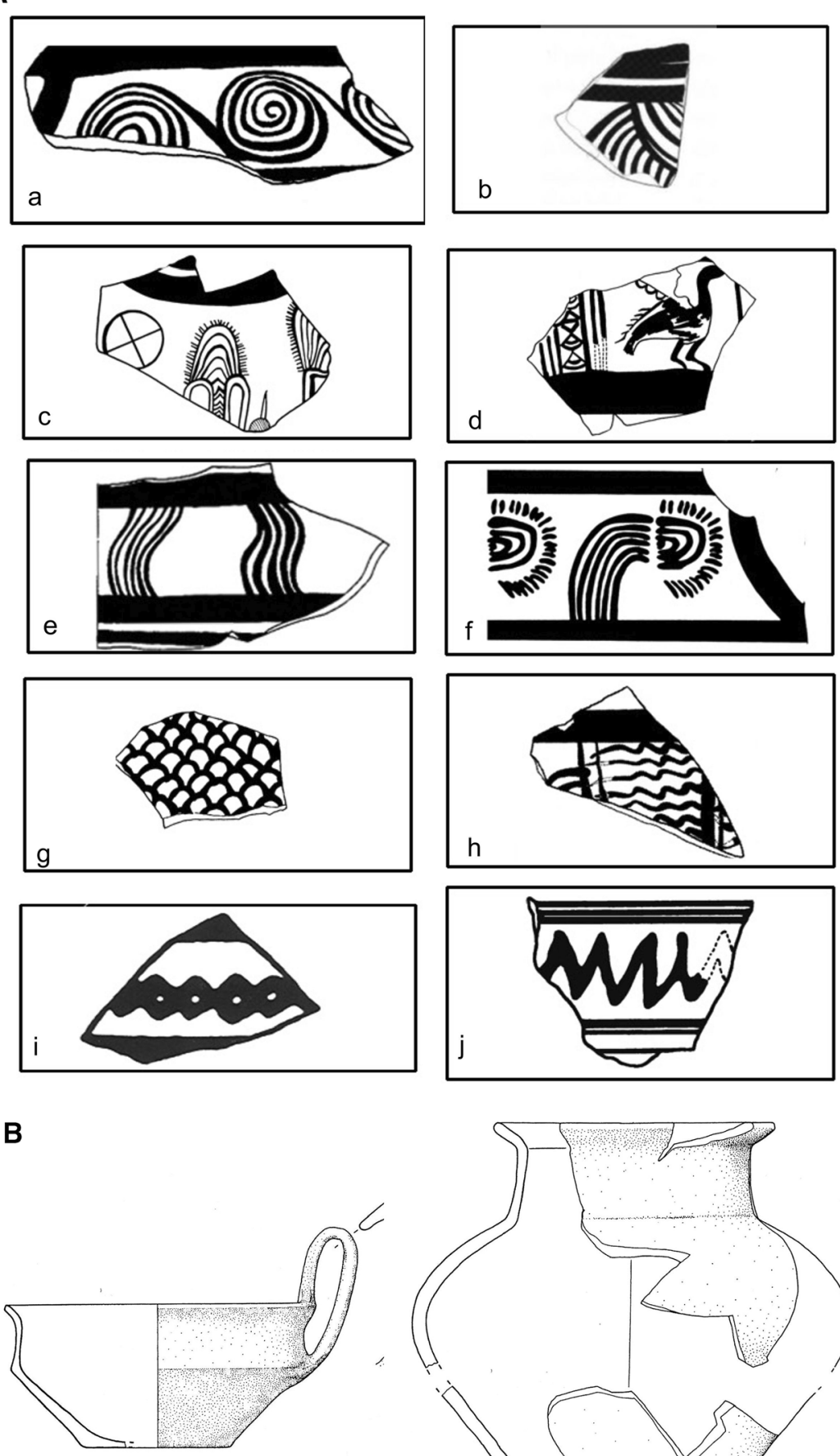

a

b
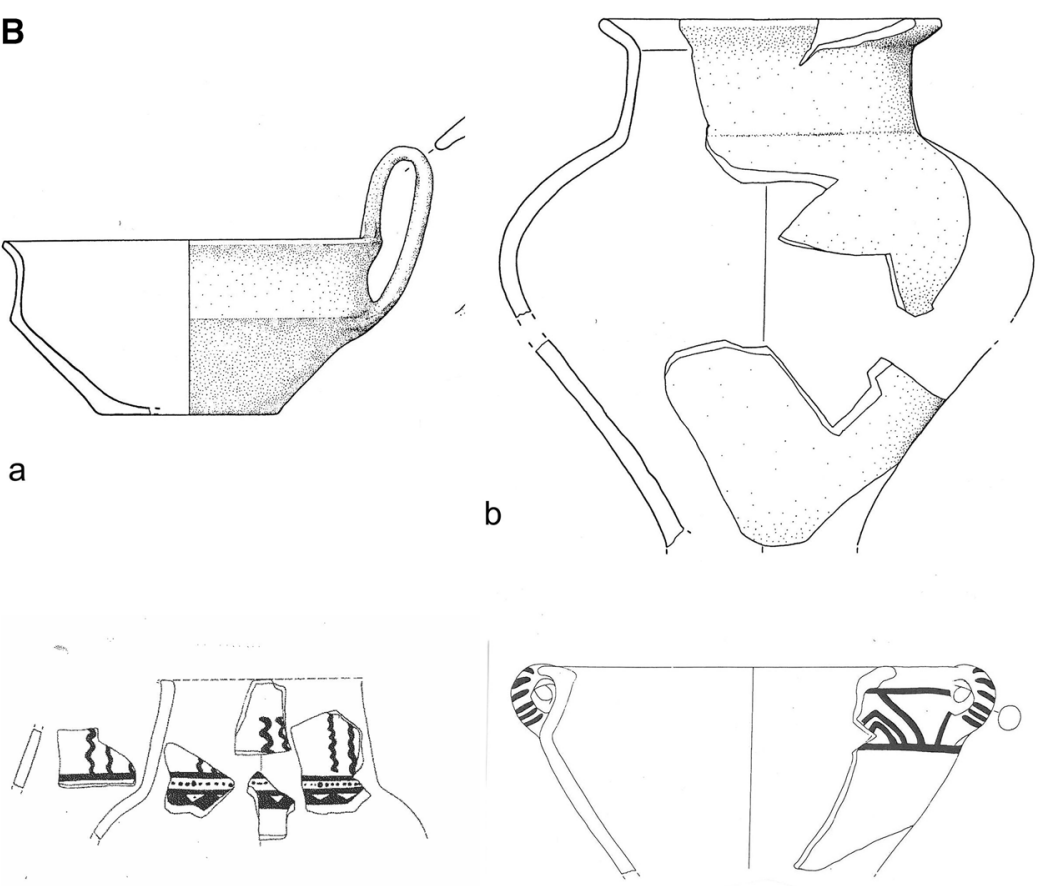

d 
Fig. 5 LH IIIB Italo-Mycenaean necked jar from Broglio di Trebisacce (sample A1, Peroni (1984, tav. 48:2)): a printed radiograph ( $R X)$, $\mathbf{b}$ drawing of the $\mathrm{RX}$, arrows show possible joints. From Levi and Cannavò in Jones et al. 2014, Fig. 5.2c, 366-367
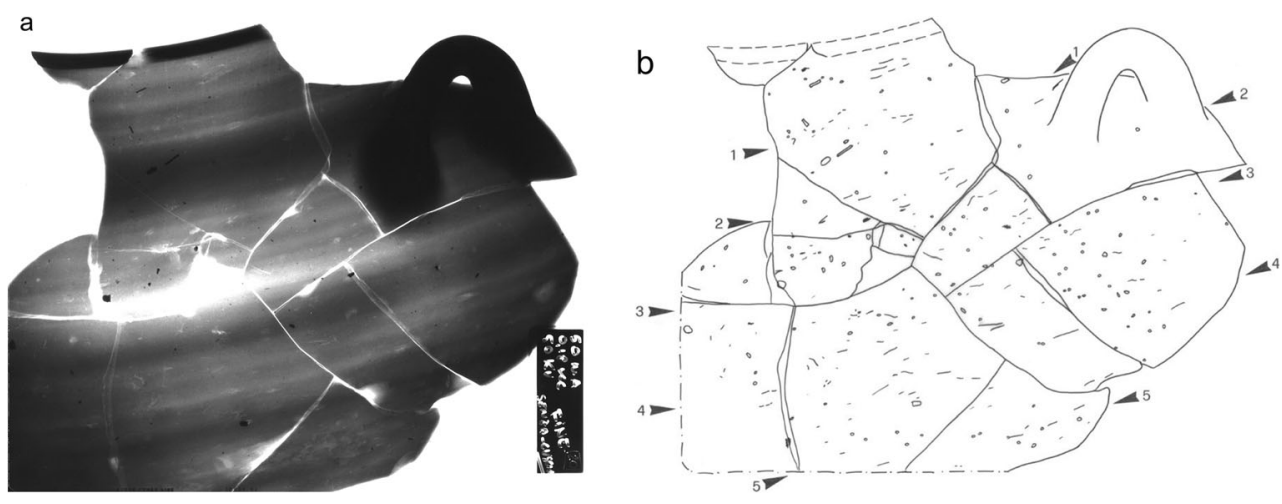

stability of the socio-economic and sociopolitical structures of those communities (Bettelli in press-a).

Two further introductory features of IM are first that this pottery was made from a fine-textured calcareous $(>5 \% \mathrm{CaO})$ clay with the assistance of the wheel. Radiographic examination of examples from Broglio di Trebisacce in Calabria (32 in Fig. 1) revealed the use of the wheel for throwing (anticlockwise) open shapes and a combination of throwing and shaping in the case of more closed shapes (Fig. 5) (Levi and Cannavò in Jones et al. 2014, 366-68).

By contrast, the contemporary indigenous pottery of coarser texture, impasto, was handmade with pressure (e.g. moulding or pinching) or with coils, although at Broglio there was some use of the wheel for finishing or even forming impasto. IM's technological similarity with decorated Aegean pottery extends to decoration in red, brown and black lustrous colours, all iron-rich and derived from clays that could only be achieved by controlled firing in a kiln. The painted decoration undoubtedly shared the same morphological and chemical characteristics as that in the imported Aegean pottery (Buxeda et al. 2003; Jones et al. 2014, 377-84), but there were some notable differences, as discussed below.
Second, Aegean ceramic influence in Italy is extended beyond the decorated pottery, IM, to hybrid (or mixed Italian) products such as (1) dolia, large storage/transport jars made from large coils of a usually tempered clay and then wheelshaped and sometimes decorated, and (2) Grey ware (Fig. 4b) made of a fine highly calcareous clay, wheel-thrown and burnished and fired under reducing conditions. In addition, there is South Italian Protogeometric and Geometric (often termed figulina) (Fig. 4b) which, while differing in shape and decoration from IM, inherits some of IM's technological characteristics, but with matt decoration, a less extensive use of the wheel and a less hard fabric due to lower firing temperatures (Jones et al. 2014, Table 1.2). There is also a further locally produced fine ware, at present only attested at Coppa Nevigata in Recent Bronze Age layers, which has a burnished surface (abbreviated BU), light colour and decorated with white paint (Vagnetti et al. 2012, 419, Fig. 12; Bettelli 2012, Fig. 1; Bettelli et al. 2017).

Assessing the status of examples of Aegean-type pottery as either 'local' (IM) or imported from the Aegean (M) was achieved by chemical analysis which was applied to nearly 500 examples from 46 sites: 276 (57\%) were identified as IM from 30 findspots. The proportion of $\mathrm{M}$ to IM varies
Fig. 6 Proportions of Aegeanimported Mycenaean (M) and Italo-Mycenaean (IM) pottery in various parts of Italy according to the chemical data (the numbers indicate the samples analysed)

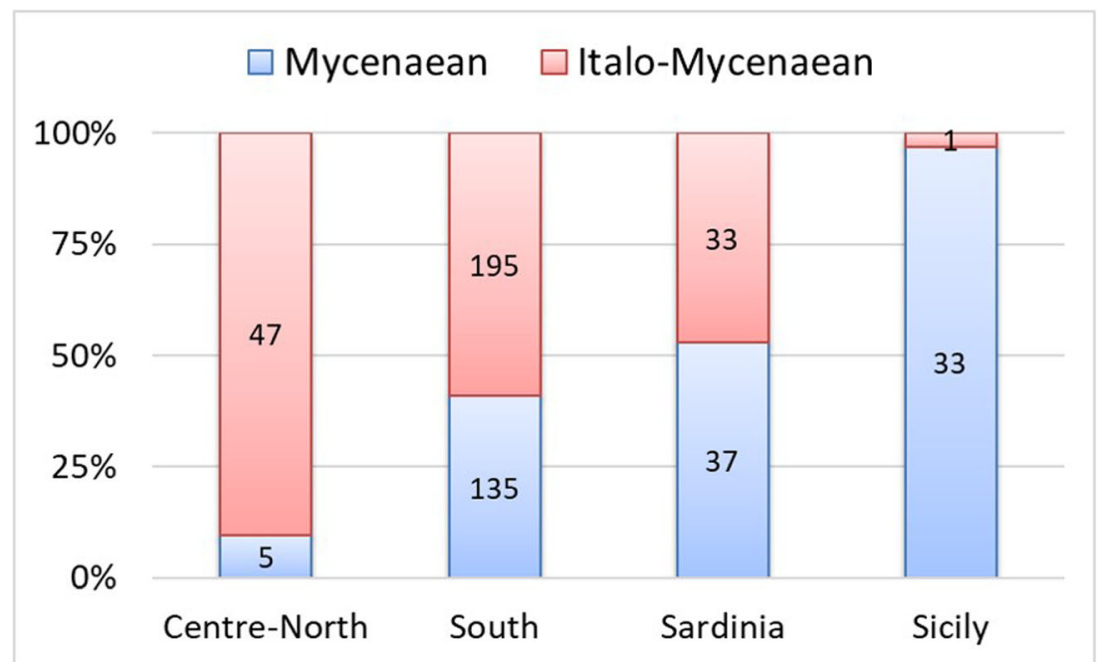




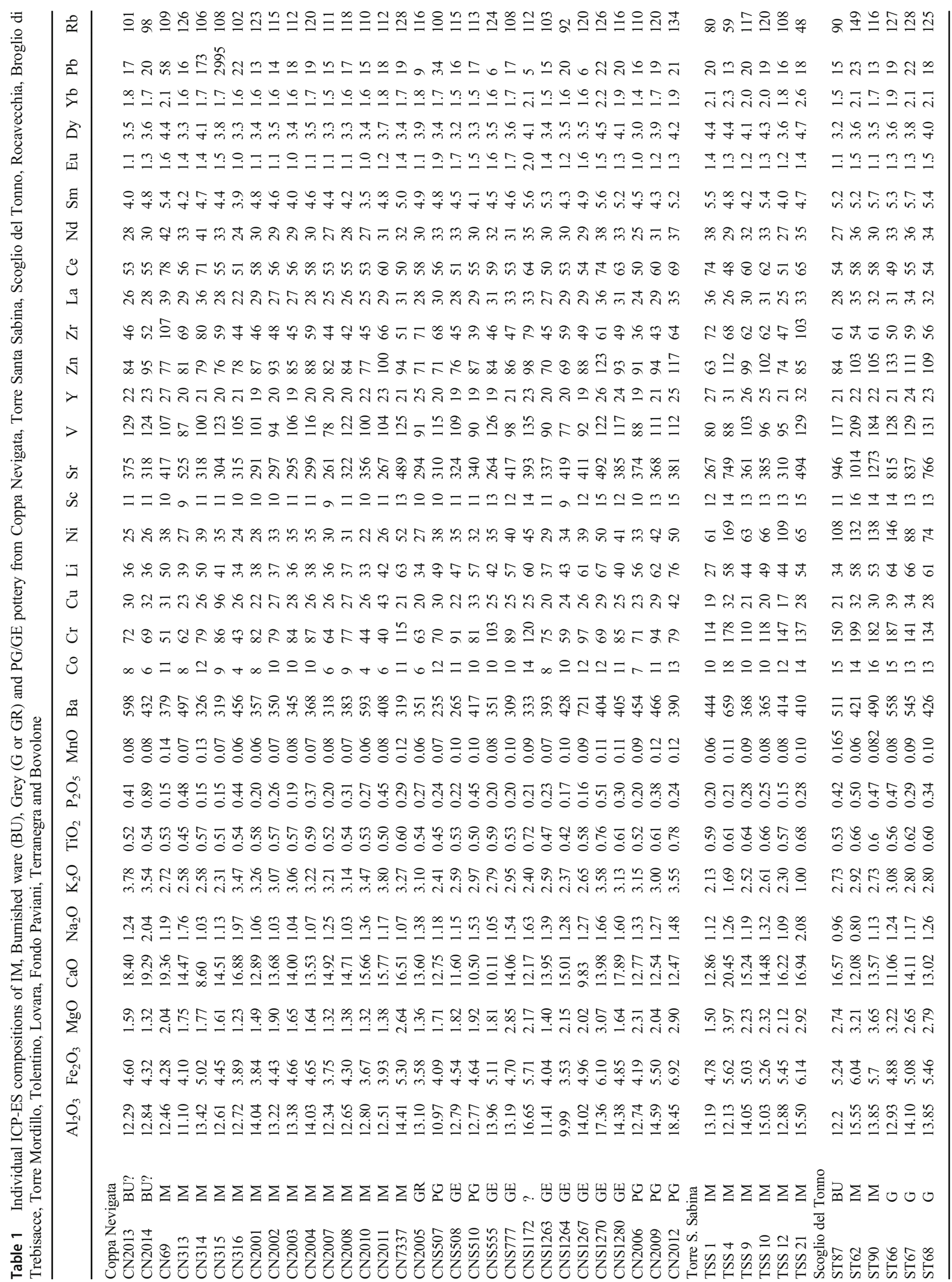




\begin{tabular}{|c|c|c|c|}
\hline थे & 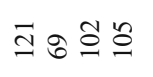 & 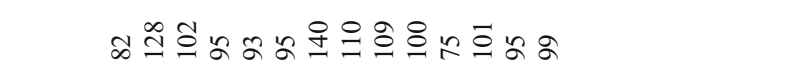 & 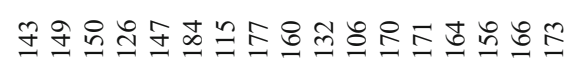 \\
\hline 2 & $26 \stackrel{2}{2}$ & 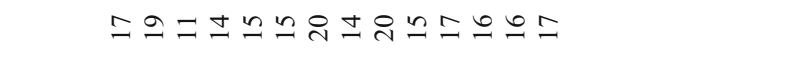 & 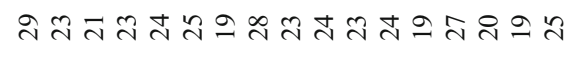 \\
\hline 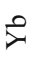 & i̊ํㅛ $\stackrel{0}{-} \stackrel{0}{-}$ & 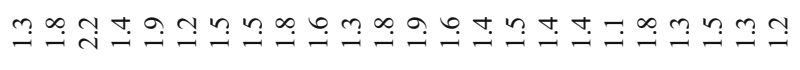 & 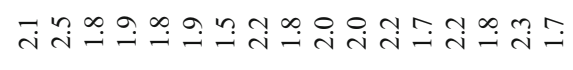 \\
\hline à & $\stackrel{+}{+}=\dot{\forall} \dot{m}$ & 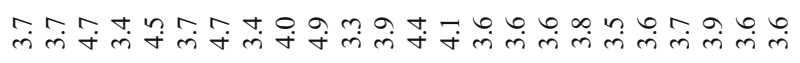 & 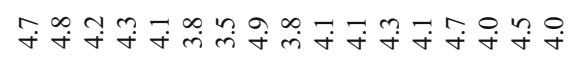 \\
\hline 武 & $\stackrel{7}{7} \mathcal{7}$ & 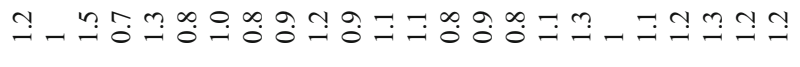 & 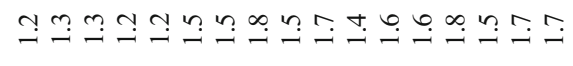 \\
\hline हี & 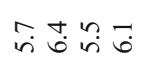 & 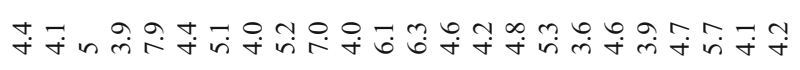 & กุ \\
\hline $\mathrm{z}$ & 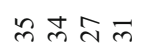 & 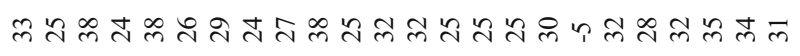 & 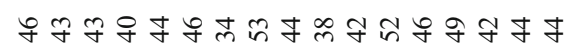 \\
\hline U & 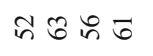 & 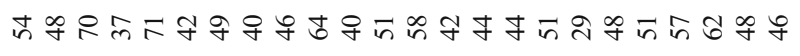 & 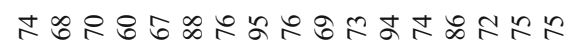 \\
\hline ב & mn் & নী ন & 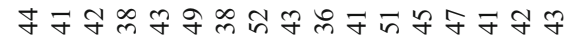 \\
\hline ง & 视苏 & 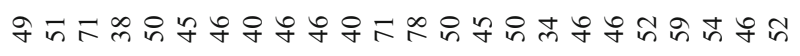 & 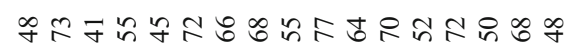 \\
\hline N & 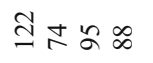 & 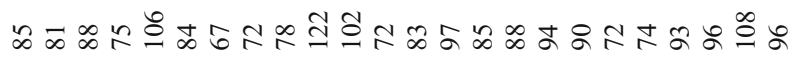 & 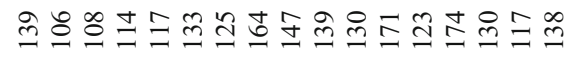 \\
\hline & $\tilde{\lambda} \check{\sim}$ & 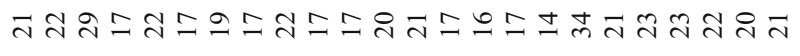 & ส่ \\
\hline & $\stackrel{g}{ \pm} \stackrel{0}{\circ}$ & 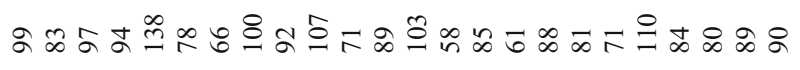 & 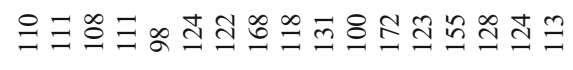 \\
\hline in & 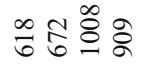 & 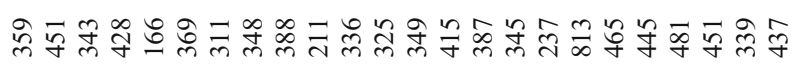 & 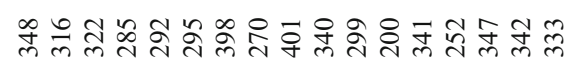 \\
\hline$\ddot{n}$ & $\ddot{M} \cong \mathscr{M}$ & 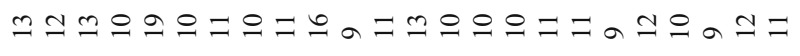 & 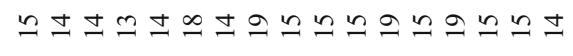 \\
\hline Z & 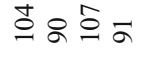 & 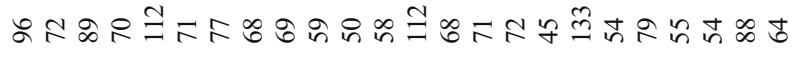 & 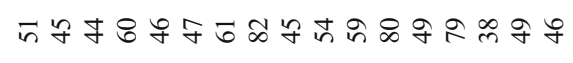 \\
\hline$\exists$ & in 氐 $q$ & 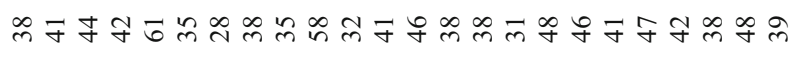 & 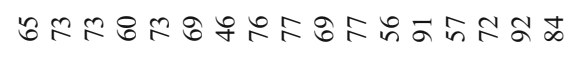 \\
\hline$\Xi$ & 永六 & 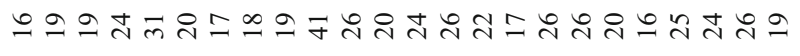 & 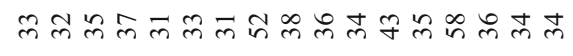 \\
\hline j & さิ & 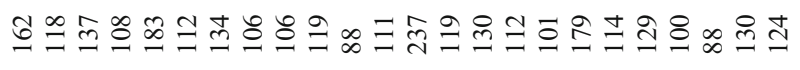 & 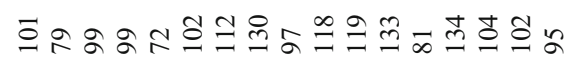 \\
\hline 8 & $\because \cong \cong \pm$ & 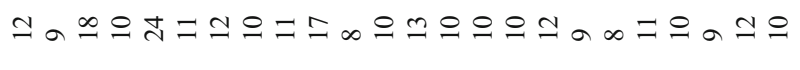 & 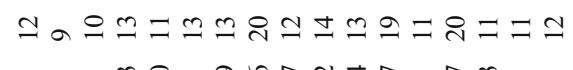 \\
\hline ص & 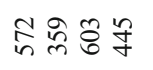 & 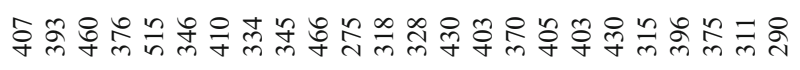 & $\stackrel{\infty}{\circ} \hat{g} \hat{\alpha}$ \\
\hline$\stackrel{0}{\xi}$ & 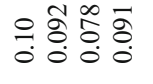 & 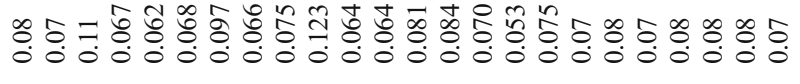 & 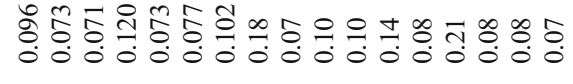 \\
\hline$\stackrel{0}{2}$ & 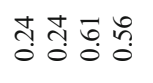 & 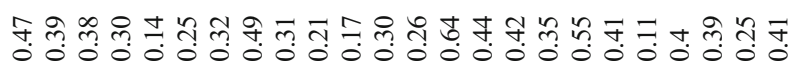 & 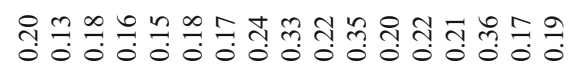 \\
\hline$\overbrace{}^{2}$ & 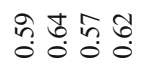 & 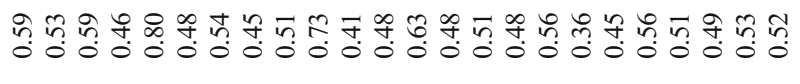 & 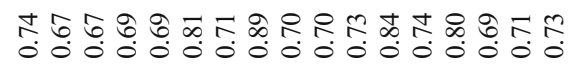 \\
\hline$\underset{\widetilde{N}}{\stackrel{0}{\sim}}$ & 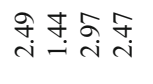 & 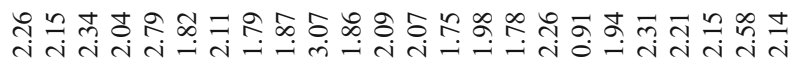 & 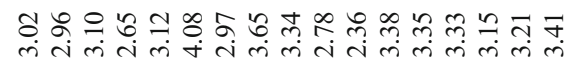 \\
\hline Z & 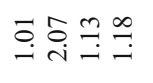 & 司 & 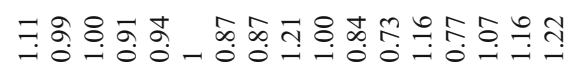 \\
\hline ల్ & 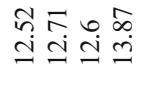 & 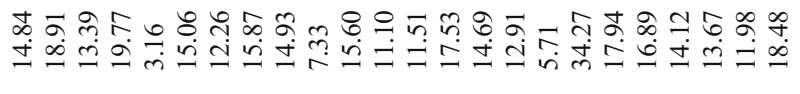 & 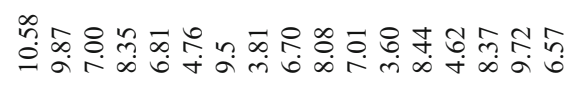 \\
\hline$\sum_{i}^{\infty}$ & 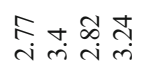 & 읏 & 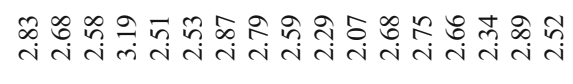 \\
\hline 今. & 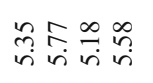 & 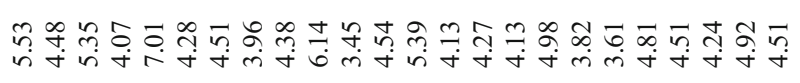 & 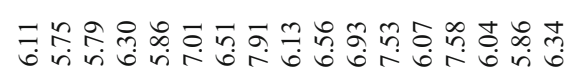 \\
\hline $0^{m}$ & 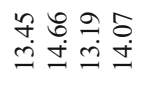 & 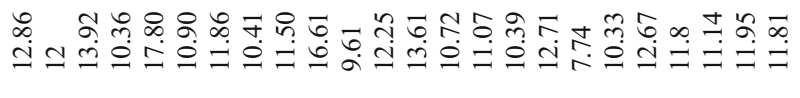 & 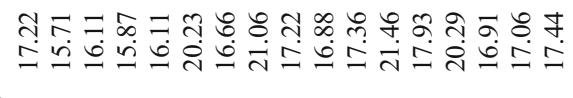 \\
\hline & & $\sim \sim \sim \sim$ & 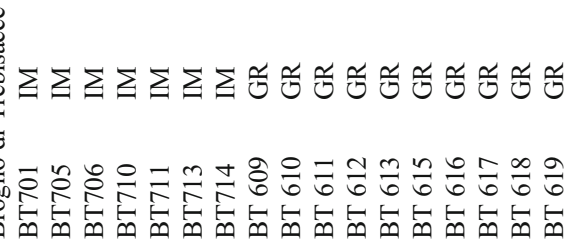 \\
\hline
\end{tabular}




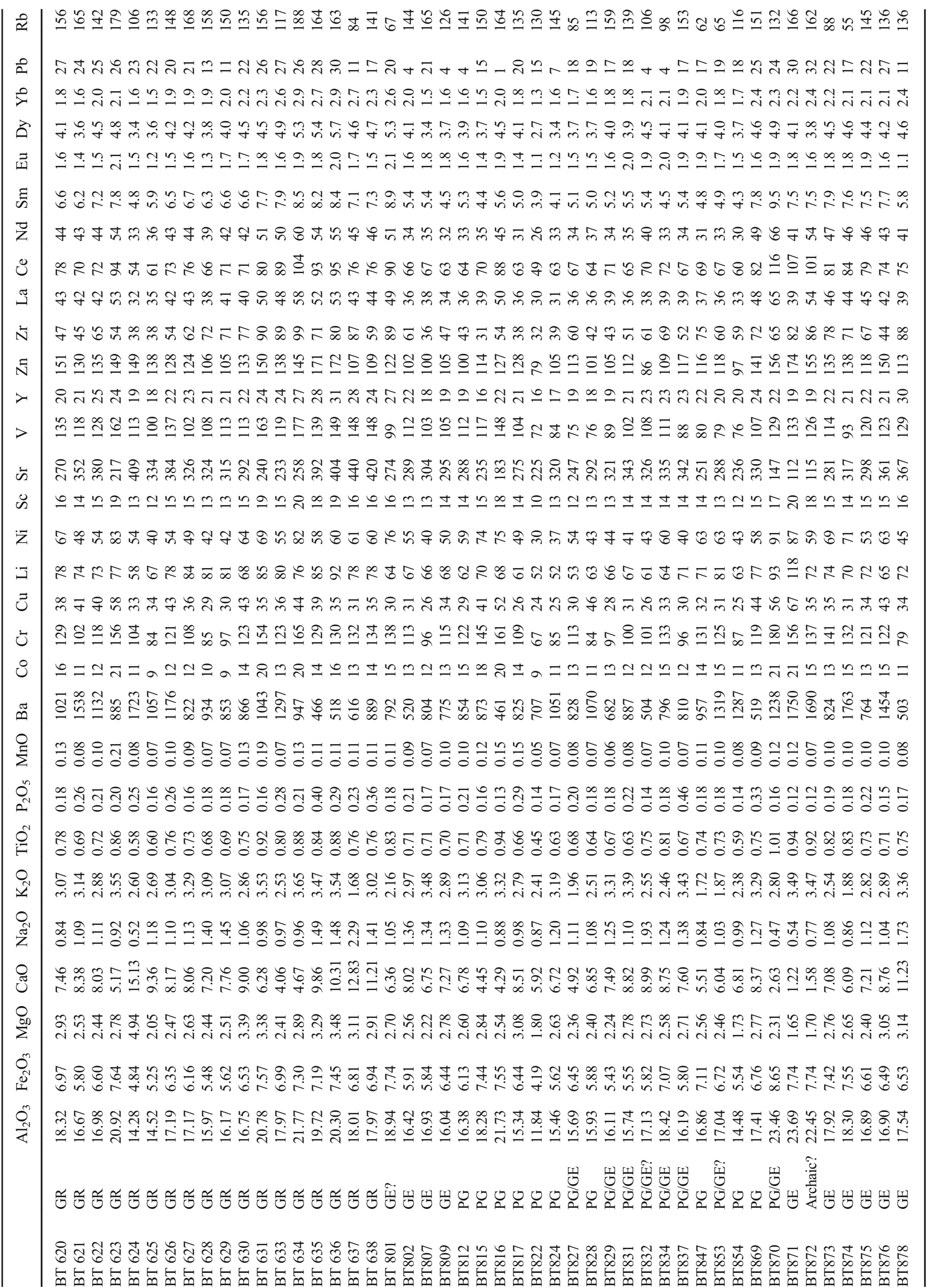




\begin{tabular}{|c|c|c|c|c|c|c|c|}
\hline थे & 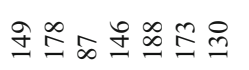 & Iิ $\cong$ & & \multicolumn{2}{|c|}{ 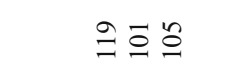 } & $\stackrel{\infty}{\simeq} \stackrel{0}{0}$ & $\exists \cong$ \\
\hline$\stackrel{2}{2}$ & 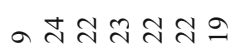 & ลิจ & 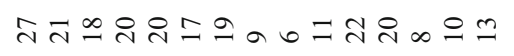 & \ัণ & $\stackrel{-}{-}$ & ते & สิ ષีત \\
\hline 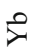 & 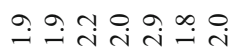 & $\cong \stackrel{\infty}{=} \stackrel{0}{-}$ & 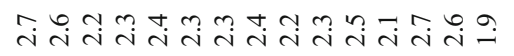 & $\hat{i} \underset{i}{i}$ & $\stackrel{\circ}{i} \hat{i}$ & $\stackrel{\circ}{i} \stackrel{0}{m}$ & 부루요 \\
\hline$\widehat{a}$ & 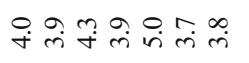 & 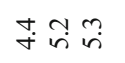 & 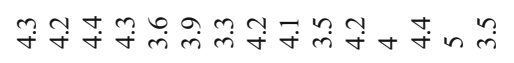 & ing in & $\stackrel{+}{+} \stackrel{+}{+}$ & 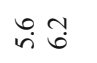 & 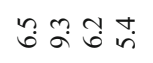 \\
\hline $\overrightarrow{\mid r}$ & 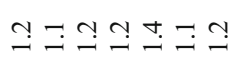 & $\stackrel{Y}{=}=\stackrel{0}{=}$ & 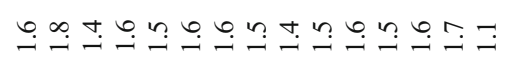 & 웅요 & $\exists a$ & $\because$ & $\stackrel{+}{\rightarrow} \stackrel{0}{-} \tilde{0}$ \\
\hline हิ & 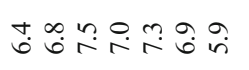 & $\ddot{n}$ तु & 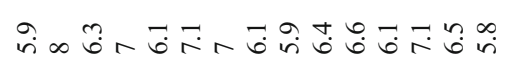 & $\stackrel{m}{n} \underset{7}{7}$ & in & $n \hat{\varrho}$ & तु \\
\hline Z & 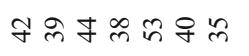 & mેळ్ & 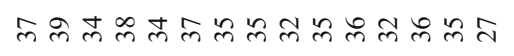 & n̄m & हे ₹ & in in & 앙ํㅆㄹㅠ \\
\hline U & 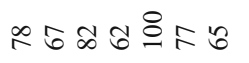 & 8 in & 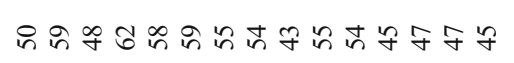 & 守 & 85 & $\stackrel{⿱ 亠 乂}{N}$ & チらケっ \\
\hline$\widetilde{J}$ & 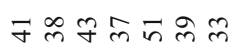 & స్లిల్ల & 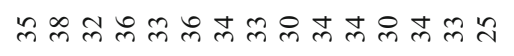 & में & $\hat{m} q$ & $n \pi$ & 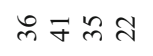 \\
\hline N & 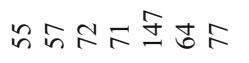 & 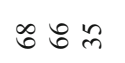 & 央 8 \&的的的品 8 品 & $\infty$ 字 & t & $\cong \cong$ & in $\infty$ ర్ర \\
\hline ธี & 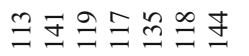 & $\stackrel{\nexists \beth \Xi}{\beth}$ & 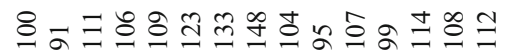 & 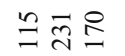 & $\alpha \bowtie$ & $\stackrel{\infty}{\infty} \underset{\infty}{\infty}$ & $\stackrel{\Xi}{\Xi \Xi}$ \\
\hline$\succ$ & 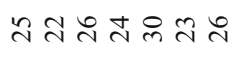 & 구누 & 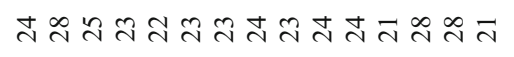 & $\ddot{n}$ 办 & $\pi \nexists$ & $\infty i$ & ำ \\
\hline$y$ & 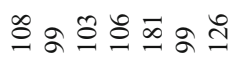 & $\bar{m} \approx \approx$ & 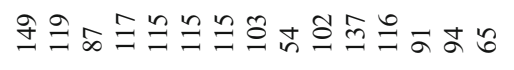 & $\hat{a} \approx \Delta$ & $\tilde{\varrho} \cong$ & $\ddot{\varrho}$ & 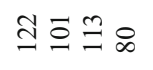 \\
\hline$\ddot{n}$ & 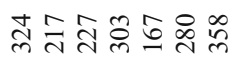 & 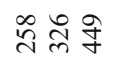 & 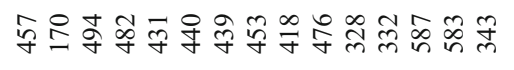 & 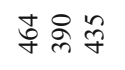 & $\approx \tilde{m}$ & 竝 & 웣ำ \\
\hline$\ddot{n}$ & 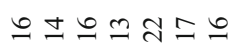 & $\simeq \pm \simeq$ & 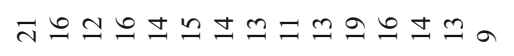 & $\pm \bigcirc \pm$ & \pm \pm & $\cong \varrho$ & $\pm ゚ \pm 0$ \\
\hline$z$ & 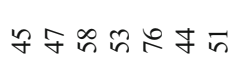 & $\stackrel{+}{a} \infty$ & 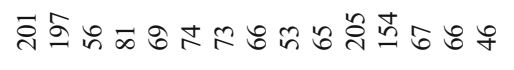 & $\infty$ in & $i n$ & $\approx \dot{n}$ & 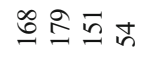 \\
\hline$\exists$ & 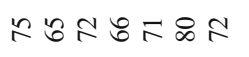 & nis & 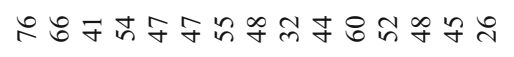 & 负合子 & 58 & F号 & 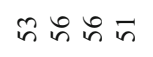 \\
\hline$\exists$ & 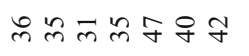 & 뉴꾜 & 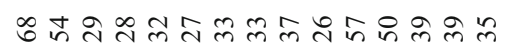 & $\ddot{m} g$ mे & 苑 & 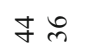 & 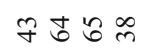 \\
\hline む & $\Re 尺 \infty \equiv \Sigma \stackrel{\varrho}{\Xi}$ & $\stackrel{\infty}{\circ} \infty$ & 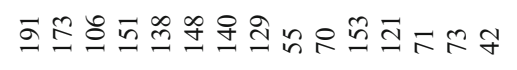 & $\stackrel{\infty}{\check{c}} \approx \tilde{\Xi}$ & $\stackrel{\infty}{\oplus} \cong$ & $\Sigma \cong$ & 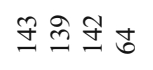 \\
\hline 8 & $=\simeq \pm \cong \pi \cong \simeq$ & $\simeq \pm \curvearrowleft$ & 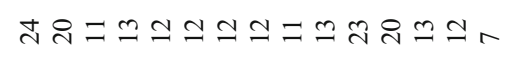 & オチ出 & \pm \pm & $\because 2$ & ๙ุণ \\
\hline$\ddot{\oplus}$ & 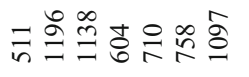 & 安専茴 & 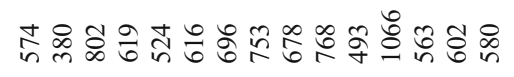 & $\infty \frac{\infty}{\infty} \hat{n}$ & go & 盯亮 & 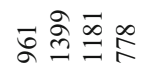 \\
\hline § & 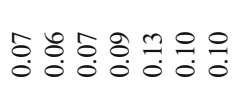 & 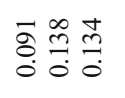 & 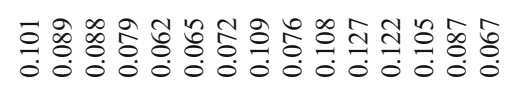 & 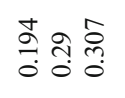 & $\frac{8}{\circ}$ & & 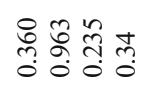 \\
\hline$\overbrace{\substack{n \\
n}}^{n}$ & 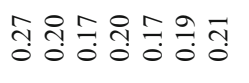 & 市芒号 & तิ & 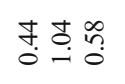 & 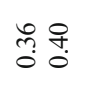 & $\cong$ 热 & 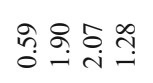 \\
\hline$\overbrace{:}^{N}$ & 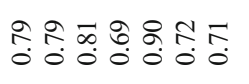 & $\begin{array}{ll}\infty & 0 \\
\infty & 0 \\
0 & 0 \\
0 & 0\end{array}$ & 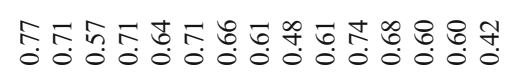 & 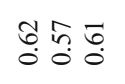 & 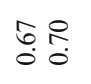 & $\stackrel{2}{0}:$ & \begin{tabular}{llll}
0 & 0 \\
\hdashline & 0 & 0 & 0 \\
0 & 0 & 0 \\
0
\end{tabular} \\
\hline 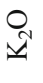 & 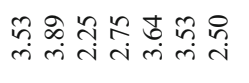 & 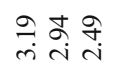 & ڤ્તિં & $\underset{\infty}{i} \underset{i}{*} \stackrel{i}{i}$ & $\underset{i}{i} \vec{i}$ & $\stackrel{\text { ते }}{\stackrel{i}{i}}$ & 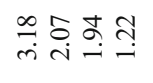 \\
\hline$\tilde{\mathbb{Z}}^{\top}$ & 車 & 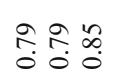 & $\begin{array}{l}0 \\
0 \\
0\end{array}$ & : & $\stackrel{9}{=}$ & & 可 승융웅 \\
\hline o & 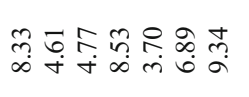 & 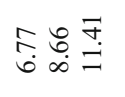 & 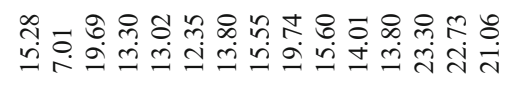 & 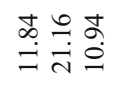 & $8:$ & مo & \\
\hline$\stackrel{O}{\infty}_{i}^{\infty}$ & 손 & 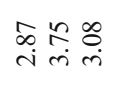 & 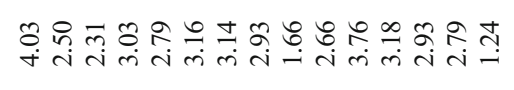 & 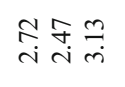 & $\stackrel{n}{i} \underset{i}{\stackrel{9}{i}}$ & $\stackrel{n}{\rightarrow} \underset{i}{n}$ & 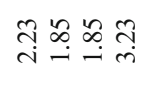 \\
\hline 0 & 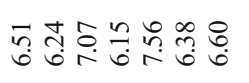 & 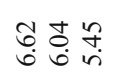 & 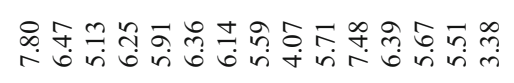 & 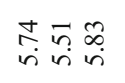 & 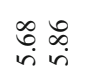 & $\stackrel{0}{0} \underset{i}{0}$ & 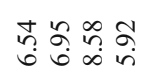 \\
\hline & 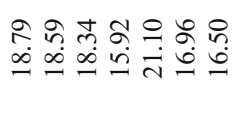 & 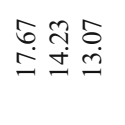 & 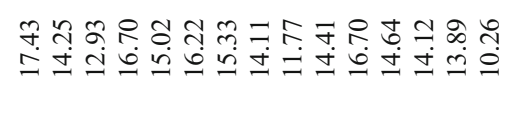 & 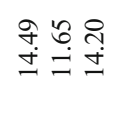 & 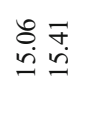 & $\delta$ & 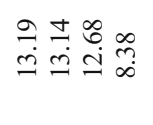 \\
\hline & 떵 똔 & 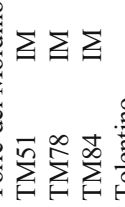 & $\geqq \geqq$ & $\sum \sum$ & & 5 & $\sum \vdots$ \\
\hline
\end{tabular}


consistently geographically: while IM forms the majority in the Centre-North (Veneto, Marche, Lazio) and South (Apulia, Basilicata, Calabria, Campania), the percentage decreases in Sardinia and almost disappears in Sicily (Fig. 6).

Since the time of our publication, new results have been reported, for instance, on finds from Rome (Bettelli 2019; Jones 2019) and from Fondo Paviani in Veneto region (Bettelli et al. 2015).

Over the long duration of the investigation, three techniques of analysis were applied to examples of IM/M as well as Grey ware, dolia, impasto, figulina and clays: AAS (10 elements; 450 samples), NAA (18 elements; 376 samples) and ICP-ES (30 elements; $c$. 400 samples). The ability to classify the compositions as either 'local' or imported rested first on the presence of large chemical databases for Late Bronze Age fine ware production centres in the relevant areas of the Aegean (Jones et al. 2014, Table 4.2, Databases 1-3) and second significant differences in certain elements between the products of some of those Aegean centres and either clays or local fine wares in Italy. Impasto from the same findspots as IM was also analysed, but its composition did not usually constitute a direct local reference because it was commonly the product of a tempered and less calcareous clay.

The archaeological implications of the extent of IM production are considerable. They include the popularity of this pottery as a mark of status among the emerging elites in Italian society at this time. They indicate the presence of potters in Italy who were proficient in the manufacture of this high quality pottery. In the early phase, such potters had most likely arrived as travellers from the Greek mainland/Aegean, many of them perhaps looking for new markets outside the Mycenaean world and responding to the demands of local (Italian) elites. In any case, they brought their potting skills with them and crucially were able to establish themselves at one or more locations in Italy, finding the appropriate clays and other materials, as well as setting up a workshop. Over the course of time, there must presumably have been interaction with local potters which led to the technology being transferred to them. But on this presumption, the Italian potter, although now trained in working on the wheel and firing in a kiln, was not bound to, or did not have the deep experience of, the canon of Mycenaean shapes and decorative motifs. The potter was free to adapt and experiment. This is important because stylistic analysis of IM points to some degree of idiosyncrasy in style and furthermore to notable regional variation, as mentioned above. At the same time, the phenomenon of technological transfer was far from comprehensive across Italy and its islands; the evidence for IM on Sicily, for example, is exceedingly sparse, as it also seems to be at a coastal mainly LH IIIC site, Punta di Zambrone, in southern Calabria (Fig. 1: 40), almost overlooking Sicily. Here, the results of NAA of Aegean-type pottery indicated almost no evidence for local IM production, the pottery coming instead principally from western Greece (Jung et al. 2015); comment on the claim of a very limited circulation of IM involving the Plain of Sybaris is given in the Discussion.

The starting point of this paper is that same issue of regionalism: was IM produced at individual sites or were there centres from which there was distribution across a region or further afield? Since these questions were only partially tackled in the 2014 publication, we examine here:

1. Inter- and intra-regional chemical differentiation of IM focusing on those sites represented by meaningful numbers of IM samples which were analysed by ICP-ES, NAA and AAS and whose compositions were published by Jones et al. (2014, Databases 1-3):

Apulia (Coppa Nevigata, Torre Santa Sabina, Scoglio del Tonno and Rocavecchia), Basilicata (Termitito), Calabria (Broglio di Trebisacce), Latium (Monte Rovello and Luni sul Mignone), Marche (Tolentino, Jesi and Ancona), Veneto (Frattesina, Lovara, Fabbrica dei Soci, Bovolone, Fondo Paviani, Castello del Tartaro and Montagnana in the Po Valley) and Sardinia (Antigori).

2. Chemical differentiation on an inter- and intra-regional basis of a range of Aegean-influenced fine wares - Grey and PG and some Iron age Geometric - at sites in Apulia (Coppa Nevigata, Scoglio del Tonno and Rocavecchia) and at Broglio di Trebisacce (28 Grey, 15 PG, 21 GE, 8 PG/GE and 1 (possible) Archaic (6th c BC) examples). The Apulian data was published in Jones et al. (2014) Database 2, and all the results for Broglio di Trebisacce are previously unpublished. The main question here is whether at a given site there was uniform production of those fine wares, or alternatively they were produced from different clays and/or at different locations within the region. Dolia are considered only peripherally because they were manufactured according to a different tradition usually involving addition of temper: their fabric can in any case be distinguished from that of the fine wares macroscopically, petrographically (see below) and chemically (e.g. at Broglio di Trebisacce, Jones et al. 2014, Fig. 4.15).

Some comments on the geological environment are necessary at the outset. Along the east coast of Italy, Apulia (and Basilicata) and Marche lie within the areas of extensive sedimentary rocks sharing similar recent and older sediments: sand, conglomerates, clays, marls, limestones and sandstones. A priori chemical differentiation of these regions may therefore be suboptimal, but here, it is salutary to examine the regions' petrographic differentiation on the basis of the composition of corresponding coarse or semi-coarse impasto-see Cannavò and Levi (2018). More encouraging chemically and petrographically is the situation in Calabria where sedimentary sediments in the northern part of the Bay of Sybaris abut 
ones of intrusive and metamorphic origin to the south (see below). And the same remark should apply elsewhere: to the northern flanks of the Po Valley, the locality and hinterland of sites in Latium and close to Antigori on Sardinia where localised outcrops of volcanic rocks occur (Levi et al. 2014).

\section{Methods}

The principal chemical data set presented in this paper was obtained by inductively coupled plasma emission spectroscopy (ICP-ES) at the Department of Earth Sciences, Royal Holloway, University of London (Jones et al. 2014, 526528). Small fragments from the selected sherds were cleaned of decoration and surface weathering and then crushed to fine powder in an agate mortar. To explore inter- and intra-regional chemical differentiations, multivariate methods - average link cluster analysis (on Z score data), principal components analysis (log transformed data) and discriminant analysis (SPSS v. 21-26) - were applied to the new ICP-ES data set and the relevant NAA and ICP-ES data sets published in the Databases 2 and 3, respectively, in Jones et al. (2014). It should be understood that at some sites (e.g. in the Taranto area and at Broglio di Trebisacce), all three techniques were applied, with few exceptions, to different sets of samples, while at others, the coverage was more restricted (e.g. Latium NAA only and Marche ICP-ES only). A limitation encountered in applying PCA was that the first two PCs usually account for no more than $50-60 \%$ of the total variation. This was mitigated by checking the classification obtained against that observed in the corresponding dendrogram from ALCA.

The chemical data is supplemented by a thin section petrography component which was applied to the material from Coppa Nevigata, Rocavecchia and Broglio di Trebisacce (Tables 4 and 5). Identifications and descriptions of inclusions were made according to the scheme outlined by Cannavò and Levi (2018) who include their criteria for the identification of grog (ibid, 11-15).

\section{Results}

Table 1 presents the individual chemical compositions of all the samples treated in this study, and Table 2 presents the composition characteristics of some groups.

\section{Inter-regional discrimination}

In the present authors' first investigation of inter-regional discrimination of IM production (Jones et al. 2014, 271-75), discriminant analysis of some relevant chemical groups indicated that IM production could be viewed as a regional phenomenon, that is, each region was apparently producing its own IM. For example, the AAS compositions of IM at Porto Perone (Apulia, Fig. 1: 25), Termitito (Basilicata, Fig. 1:30) and Broglio di Trebisacce (Calabria, Fig. 1: 32) treated as three separate groups could be discriminated confidently (Jones et al. 2014, Fig. 4.40a), and, as should be expected on geological grounds, these three groups could be differentiated from the Antigori group on Sardinia (Jones et al. 2014, Fig. 4.40b). But at the same time, this process of discriminating between regional production was partly undermined by the variation in composition on an intra-group basis. A case in point is the eastern Po Valley where the relative lack of compositional uniformity was apparent in treating the NAA data for likely IM from Fondo Paviani, Frattesina, Montagnana, Fabbrica dei Soci and Castello del Tartaro (Jones et al. 2014, Fig. 4.21; here Fig. 1: 64, 61, 68, 63 and 65) and again is apparent in the ICP-ES data (Fig. 7) for IM from Lovara, Bovolone as well as Fondo Paviani (Fig. 1 for locations). We return to this data set below, while noting in the meantime that chemical and petrographic investigations by other researchers of prehistoric and later pottery of different classes as well as modern clays have already amply demonstrated the natural range of compositions existing in this region (Cannavò and Levi 2018; Picon 2000; Jones et al. 2014, 212-221; Saracino et al. 2018).

The present study has therefore demanded a change of strategy, first to classify the individual compositions using PCA and ALCA rather than working from predetermined groups; second, to introduce a necessary petrographic component that was largely missing from earlier work; and third, to move away from treating sites according to their present-day administrative region to zones that link sites according to communication by sea (Table 3 ). Such a move would take cognisance of the shared features of technique and style of IM observed within those zones (Jones et al. 2014, 455-56). The issue of the location of sites with respect to the coast is important as it acknowledges the emergence of more frequent communication by sea in Italy by the middle of the second millennium BC; long-distance maritime travel, common enough in the eastern Mediterranean at this time, was previously almost unknown in the central Mediterranean (Broodbank 2013, Figs. 7.1, 8.3, 8.54).

The zones defined here partially reflect the different lithologies locally represented in the complex geological environment of the Italian Peninsula and the main Islands. For instance, the Adriatic and Ionian zones are mainly characterised by sedimentary rocks, while the Tyrrhenian zone and Sicily frequently feature other geological components (Table 4).

Using this approach, we start with the strong support obtained by PCA and ALCA for site-specific IM production at Coppa Nevigata (Adriatic), Porto Perone, Torre Castelluccia and Broglio di Trebisacce (Ionian) and regional production within the Tyrrhenian zone at Luni (Fig. 8). The trend in this 


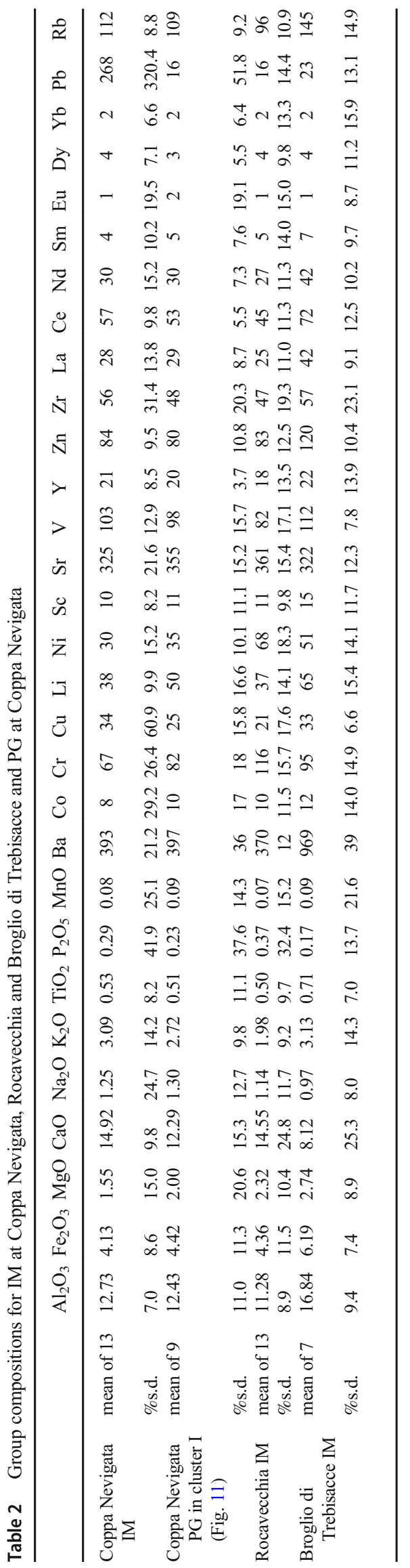

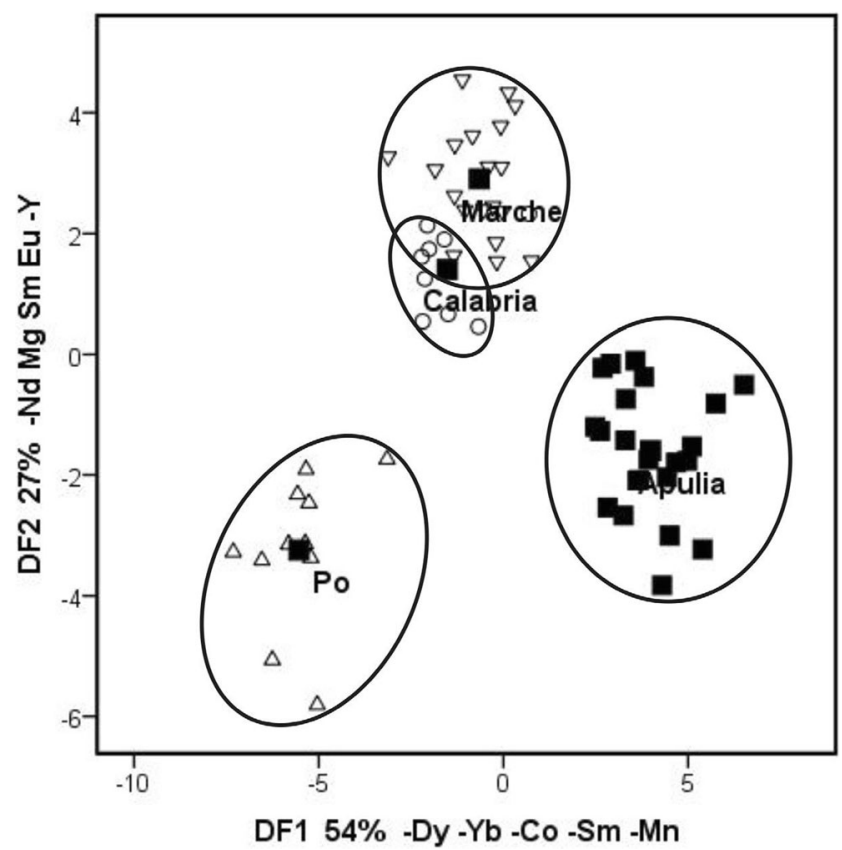

Fig. 7 DA plot of the groups of IM representing Apulia (Coppa Nevigata and Torre Santa Sabina) (匹), Calabria (Broglio di Trebisacce o), Marche (Tolentino, Jesi and Ancona $\nabla$ ) and Veneto (Lovara, Bovolone and Fondo Paviani in the Po Valley $\triangle$ ). ICP-ES data, log transformed (Jones et al. 2014, Fig. 4.42)

figure towards higher $\mathrm{Cr}$ contents in the Eastern Ionian and, more specifically, in the Taranto area (Porto Perone and Torre Castelluccia) is reflected in the corresponding data from PIXE-PIGME analysis of the fifth and fourth century BC Apulian red figure pottery; Grave et al. (1996-97) found higher $\mathrm{Cr}, \mathrm{Co}$, Ni and $\mathrm{Sr}$ contents in the Taranto area than at Ruvo and Canosa to the north.

\section{Middle-southern Adriatic}

These two zones are important for the wealth of Aegean-type pottery finds from several excavated sites in present-day Apulia, and furthermore adequate numbers of IM examples have been analysed by ICP-ES. Two main groupings of IM (observed in ALCA) are evident in the PC plot (Fig. 9), one comprising examples from Rocavecchia (I) and the other from Coppa Nevigata (II); the differences between these groupings are small except in $\mathrm{Mg}, \mathrm{K}, \mathrm{Cr}$ and $\mathrm{Ni}$ (Table 2). The IM from Torre Santa Sabina is the least uniform: one belongs to group I (TSS 12), three examples (TSS 1, 9 and 10) are close to three atypical Coppa Nevigata examples (CN 69, 314 and 7337) in group III, and TSS 4 and 21 are outliers. Figure 9 is relevant in connection with the classification of IM and the two derivatives, GR and PG/GE, from the same sites treated below (Fig. 14). CN 315 has an anomalously high $\mathrm{Pb}$ content, owing presumably to its proximity to a lead artefact.

Considering now the Middle Adriatic zone on its own, the IM compositions at Coppa Nevigata $(\mathrm{CN})$ and Tolentino are 
Table 3 Locations of sites with Italo-Aegean pottery, according to the tradition of how the pottery was made (Techniques) and to the pottery's stylistic (both morphological and decorative) aspects (adapted from Jones et al. 2014, Table 6.6)

\begin{tabular}{|c|c|c|c|c|}
\hline Zone & Sites & Techniques & Shapes & Decoration \\
\hline $\begin{array}{l}\text { Northern Adriatic/ } \\
\text { Po Valley }\end{array}$ & Lovara, Bovolone, Fondo Paviani & Aegean & Aegean & Aegean and Aegean reinterpreted \\
\hline Middle Adriatic & Tolentino & Aegean & Aegean & $\begin{array}{l}\text { Aegean and Aegean } \\
\text { reinterpreted; good quality }\end{array}$ \\
\hline Southern Adriatic & $\begin{array}{l}\text { Coppa Nevigata, Rocavecchia, } \\
\text { Leuca }\left(39^{\circ} 47^{\prime} 36.4^{\prime \prime}, 18^{\circ} 22^{\prime} 00.8^{\prime \prime}\right) \text { ? }\end{array}$ & Aegean & Aegean & Aegean; good quality \\
\hline Ionian & $\begin{array}{l}\text { Broglio di Trebisacce, Termitito, Porto Perone, } \\
\text { Scoglio del Tonno, Torre Castelluccia }\end{array}$ & Aegean & $\begin{array}{l}\text { Aegean and } \\
\text { Italian }\end{array}$ & $\begin{array}{l}\text { Aegean (with pictorial) and } \\
\text { Aegean reinterpreted; } \\
\text { good quality }\end{array}$ \\
\hline Tyrrhenian & $\begin{array}{l}\text { Luni sul Mignone, Monte Rovello, } \\
\text { Casale Nuovo }\end{array}$ & Aegean & Aegean & Aegean and Aegean reinterpreted \\
\hline Sardinia & Antigori & $\begin{array}{l}\text { Coarse raw materials, } \\
\text { inferior Aegean }\end{array}$ & Aegean & Aegean; low quality \\
\hline
\end{tabular}

treated (Table 1). Tolentino was selected from Marche because it is the site with the largest number of IM samples analysed. Figure 10 shows all but three $\mathrm{CN}$ samples (already identified as atypical in Fig. 8) forming a group of its own, separating from several smaller groupings making up the IM from Tolentino. The relative lack of chemical consistency among the latter samples, which is reflected in the comparable situation at Torre Santa Sabina (Fig. 10), may be a matter of different workshops operating in the same area, sharing similar style but different fabrics. The two samples of T13 belong to a distinctly larger vessel than the others from Tolentino.

\section{Western Ionian: Bay of Sybaris}

This area holds pride of place in this section because it was the outcome of petrographic (and to a lesser extent chemical) analysis of material from here that first exposed the extent of intra-regional movement of LBA/FBA pottery anywhere in Italy. The pottery of concern was not IM but dolia and impasto, much of it found at Broglio di Trebisacce, the site (Fig. 1: 32) that has received the fullest attention of characterisation studies of any late prehistoric/protohistoric site in Italy, ${ }^{1}$ as can be judged from the programmes of analysis presented by Jones et al. (2014, Table 4.7a).

Exploiting the underlying geological variability within the Bay, Levi (1999, Fig. 27) established that the products of the Bay's northern zone where Broglio di Trebisacce is located were characterised by sedimentary inclusions (sandstone, siltstone, calcite), whereas igneous (granite, pyroxene) and metamorphic inclusions are featured to the south (Cannavò and Levi 2018, Fig. 12). This differentiation also had a chemical dimension with $\mathrm{Cs}, \mathrm{Rb}$ and $\mathrm{K}$ being enriched in the northern

\footnotetext{
${ }^{1}$ Excluding Aeolian Islands, where the characterisation is mainly petrographic (Williams 2018; Levi et al. 2019)
}

clays and temper (siltstone and shale) and $\mathrm{Na}$ in the south (see Jones et al. 2014, Fig. 4.18). It was possible, then, to establish that a significant proportion (30 and $20 \%$ during the RBA and FBA, respectively) of dolia found at Broglio were imported from the southern part of the Plain/Bay. The corresponding figure for impasto was much lower (1-2\%).

Returning to IM, it proved possible to differentiate among the many examples of decorated Aegean-type pottery found at Broglio di Trebisacce and Torre Mordillo analysed by AAS between IM on the one hand and Aegean imports on the other (Jones et al. 2014, Fig. 4.15-4.16). Furthermore, owing to the contrasting geological environments at the two adjacent sites, the IM products were found to be capable of some inter-site discrimination, a result that resonates with the pottery's morphological and decorative attributes. Late Minoan (LM) decorative elements at Broglio and Torre Mordillo are differently organised in combination with the shapes (Fig. 4f). At the former site, they are attested mainly on closed shapes, generally the necked jars typical of this zone (Vagnetti 1984, tav. 46.3, 48; Vagnetti and Panichelli 1994, tav. 76.1). By contrast, at Torre Mordillo, they are also largely represented on open forms of Cretan legacy such as cups or bowls, consistently with the LM stylistic repertoire (Vagnetti 2001, Figs. 94.1, 95.39, 98.97, 99.106, 100.124). These differences between the two sites suggest the existence of several workshops operating in, or for, the two communities, even if within the framework of a shared taste (Bettelli and Levi in press). In summary, the archaeometric evidence for localised production of dolia in the Bay of Sybaris, already strong, is extending also to IM. That view is in any case supported by first the results leading to Fig. 8 above and second the ICP-ES compositions of IM from Broglio and two sites in the Adriatic zone (Coppa Nevigata and Rocavecchia); the latter are differentiated from Broglio in $\mathrm{Al}, \mathrm{Fe}, \mathrm{Mg}, \mathrm{Ca}$, Sc and rare earth elements (Table 2). The corresponding petrographic differences, 
Table 4 Sites with IM finds arranged by geographical zones with indication of (a) the sites' DMS coordinates and (b) the main local geological characteristics according to the Geological Map of Italy (scale 1:500.000)

\begin{tabular}{|c|c|c|c|c|}
\hline Zone & $\begin{array}{l}\text { Present-day } \\
\text { administrative } \\
\text { region }\end{array}$ & Site & $\begin{array}{l}\text { Site } \\
\text { number }\end{array}$ & Lithology \\
\hline \multirow{7}{*}{$\begin{array}{l}\text { Northern } \\
\text { Adriatic/ } \\
\text { Po } \\
\text { Valley }\end{array}$} & \multirow[t]{7}{*}{ Veneto } & $\begin{array}{l}\text { Bovolone }\left(45^{\circ} 14^{\prime} 59,34^{\prime \prime}\right. \\
\left.\quad 11^{\circ} 06^{\prime} 36,66^{\prime \prime}\right)\end{array}$ & 67 & $\begin{array}{l}\text { Sedimentary: sands, sandstones and } \\
\text { conglomerates (terraced alluvial deposits) }\end{array}$ \\
\hline & & $\begin{array}{l}\text { Montagnana }\left(45^{\circ} 13^{\prime} 50,03^{\prime \prime}\right. \\
\left.11^{\circ} 28^{\prime} 34,89^{\prime \prime}\right)\end{array}$ & 68 & $\begin{array}{l}\text { Sedimentary: sands, sandstones and } \\
\text { conglomerates (alluvial deposits of the } \\
\text { River Adige), limestones, marls; Volcanic: } \\
\text { basalts, trachytes, andesites }\end{array}$ \\
\hline & & $\begin{array}{l}\text { Castello del Tartaro }\left(45^{\circ} 06^{\prime} 57,57^{\prime \prime} \text {, }\right. \\
\left.11^{\circ} 13^{\prime} 38,12^{\prime \prime}\right)\end{array}$ & 65 & \multirow{5}{*}{$\begin{array}{l}\text { Sedimentary: sands, sandstones and } \\
\text { conglomerates (alluvial deposits of the } \\
\text { Rivers Po and Adige) }\end{array}$} \\
\hline & & $\begin{array}{l}\text { Fondo Paviani ( } 45^{\circ} 07^{\prime} 16,39^{\prime \prime} \\
\left.11^{\circ} 17^{\prime} 26,77^{\prime \prime}\right)\end{array}$ & 64 & \\
\hline & & $\begin{array}{l}\text { Fabbrica dei Soci }\left(45^{\circ} 05^{\prime} 10,06^{\prime \prime}\right. \\
\left.11^{\circ} 20^{\prime} 10,07^{\prime \prime}\right)\end{array}$ & 63 & \\
\hline & & Lovara $\left(45^{\circ} 08^{\prime} 59,98^{\prime \prime}, 11^{\circ} 22^{\prime} 19,85^{\prime \prime}\right)$ & 62 & \\
\hline & & Frattesina $\left(45^{\circ} 01^{\prime} 19,56^{\prime \prime}, 11^{\circ} 38^{\prime} 46,92^{\prime \prime}\right)$ & 61 & \\
\hline \multirow[t]{7}{*}{ Adriatic } & \multirow[t]{3}{*}{ Marche } & $\begin{array}{l}\text { Ancona (Montagnolo) }\left(43^{\circ} 37^{\prime} 1,2^{\prime \prime} \text {, }\right. \\
\left.13^{\circ} 30^{\prime} 28,3^{\prime \prime}\right)\end{array}$ & 60 & $\begin{array}{l}\text { Sedimentary: marls, calcareous marls, clays, } \\
\text { limestones, sandstones, conglomerates, sands, } \\
\text { evaporites }\end{array}$ \\
\hline & & 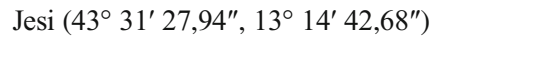 & 59 & $\begin{array}{l}\text { Sedimentary: sands, conglomerates, clays, } \\
\text { marls, limestones }\end{array}$ \\
\hline & & Tolentino $\left(43^{\circ} 13^{\prime} 21,12^{\prime \prime}, 13^{\circ} 20^{\prime} 1,9^{\prime \prime}\right)$ & 58 & Sedimentary: sandstones, clays, marls \\
\hline & \multirow[t]{4}{*}{ Apulia } & $\begin{array}{l}\text { Coppa Nevigata }\left(41^{\circ} 33^{\prime} 28,86^{\prime \prime}, 15^{\circ} 50^{\prime}\right. \\
\left.1,52^{\prime \prime}\right)\end{array}$ & 3 & Sedimentary: limestones, clays, sands \\
\hline & & $\begin{array}{l}\text { Torre S. Sabina - C. morelli } \\
\left(40^{\circ} 45^{\prime} 36,94^{\prime \prime}\right. \\
\left.17^{\circ} 41^{\prime} 43,79^{\prime \prime}\right)\end{array}$ & 13 & Sedimentary: limestones, sands \\
\hline & & $\begin{array}{l}\text { Punta le Terrare }\left(40^{\circ} 38^{\prime} 43,73^{\prime \prime}, 17^{\circ} 57^{\prime}\right. \\
\left.32,86^{\prime \prime}\right)\end{array}$ & 16 & Sedimentary: clays, sandstones, limestones, sands \\
\hline & & $\begin{array}{l}\text { Rocavecchia }\left(40^{\circ} 17^{\prime} 14,92^{\prime \prime}\right. \\
\left.18^{\circ} 25^{\prime} 36,4^{\prime \prime}\right)\end{array}$ & 17 & $\begin{array}{l}\text { Sedimentary: limestones, clays, marls, } \\
\text { conglomerates, sands }\end{array}$ \\
\hline \multirow[t]{7}{*}{ Ionian } & \multirow[t]{3}{*}{ Apulia } & $\begin{array}{c}\text { Torre Castelluccia }\left(40^{\circ} 20^{\prime}\right. \\
\left.33,44^{\prime \prime}, 17^{\circ} 22^{\prime} 53,59^{\prime \prime}\right)\end{array}$ & 24 & Sedimentary: limestones, clays, marls, sands \\
\hline & & $\begin{array}{l}\text { Porto Perone }\left(40^{\circ} 22^{\prime} 19,97^{\prime \prime}\right. \\
\left.17^{\circ} 18^{\prime} 29,02^{\prime \prime}\right)\end{array}$ & 25 & Sedimentary: limestones, marls, clays \\
\hline & & $\begin{array}{l}\text { Scoglio del Tonno }\left(40^{\circ} 29^{\prime}\right. \\
\left.0,59^{\prime \prime}, 17^{\circ} 13^{\prime} 31,99^{\prime \prime}\right)\end{array}$ & 26 & Sedimentary: limestones, marls, clays, conglomerates \\
\hline & \multirow[t]{2}{*}{ Basilicata } & S. Vito di Pisticci $\left(40^{\circ} 23^{\prime} 30^{\prime \prime}, 16^{\circ} 33^{\prime} 31,68^{\prime \prime}\right)$ & 29 & $\begin{array}{l}\text { Sedimentary: sands, limestones, } \\
\text { conglomerates, clays, marls }\end{array}$ \\
\hline & & Termitito $\left(40^{\circ} 13^{\prime} 13,1^{\prime \prime}, 16^{\circ} 40^{\prime} 6,26^{\prime \prime}\right)$ & 30 & $\begin{array}{l}\text { Sedimentary: clays, conglomerates, sands, } \\
\text { sandstones, radiolarites }\end{array}$ \\
\hline & \multirow[t]{2}{*}{ Calabria } & $\begin{array}{l}\text { Broglio di Trebisacce } \\
\qquad\left(39^{\circ} 51^{\prime} 51,16^{\prime \prime}, 16^{\circ} 30^{\prime} 17,25^{\prime \prime}\right)\end{array}$ & 32 & $\begin{array}{l}\text { Sedimentary: sandstones, limestones, } \\
\text { conglomerates, clays, sands, chert }\end{array}$ \\
\hline & & $\begin{array}{l}\text { Torre Mordillo }\left(39^{\circ} 42^{\prime} 45,72^{\prime \prime}, 16^{\circ} 18^{\prime}\right. \\
\left.\quad 42,56^{\prime \prime}\right)\end{array}$ & 34 & $\begin{array}{l}\text { Sedimentary: sands, conglomerates, sandstones, } \\
\text { limestones, clays; Metamorphic: phyllites, gneisses }\end{array}$ \\
\hline \multirow[t]{5}{*}{ Tyrrhenian } & \multirow[t]{3}{*}{ Latium } & $\begin{array}{l}\text { Luni sul Mignone }\left(42^{\circ} 13^{\prime}\right. \\
\left.\quad 35,34^{\prime \prime}, 11^{\circ} 56^{\prime} 6,67^{\prime \prime}\right)\end{array}$ & 54 & $\begin{array}{l}\text { Sedimentary: marls, clays, sands, conglomerates; } \\
\text { Volcanic: rhyolites, trachytes, latites, phonolites }\end{array}$ \\
\hline & & $\begin{array}{l}\text { Monte Rovello ( } 42^{\circ} 09^{\prime} 34,28^{\prime \prime} \text {, } \\
\left.11^{\circ} 53^{\prime} 9,93^{\prime \prime}\right)\end{array}$ & 53 & $\begin{array}{l}\text { Sedimentary: marls, clays, sands, limestones; } \\
\text { Volcanic: rhyolites }\end{array}$ \\
\hline & & $\begin{array}{l}\text { Casale Nuovo }\left(41^{\circ} 27^{\prime} 38,66^{\prime \prime}\right. \\
\left.12^{\circ} 49^{\prime} 10,2^{\prime \prime}\right)\end{array}$ & 51 & $\begin{array}{l}\text { Sedimentary: sands, clays; Volcanic: } \\
\text { tephrites, foidites }\end{array}$ \\
\hline & Molise & $\begin{array}{l}\text { Monteroduni }\left(41^{\circ} 31^{\prime} 52,77^{\prime \prime}\right. \\
\left.14^{\circ} 08^{\prime} 36,53^{\prime \prime}\right)\end{array}$ & 50 & Sedimentary: limestone, marls \\
\hline & Campania & $\begin{array}{l}\text { Pontecagnano }\left(40^{\circ} 38^{\prime} 46,78^{\prime \prime}\right. \\
\left.14^{\circ} 53^{\prime} 0,19^{\prime \prime}\right)\end{array}$ & 46 & $\begin{array}{l}\text { Sedimentary: travertines, sands, conglomerates, } \\
\text { shales, limestone }\end{array}$ \\
\hline
\end{tabular}


Table 4 (continued)

\begin{tabular}{|c|c|c|c|c|}
\hline Zone & $\begin{array}{l}\text { Present-day } \\
\text { administrative } \\
\text { region }\end{array}$ & Site & $\begin{array}{l}\text { Site } \\
\text { number }\end{array}$ & Lithology \\
\hline & Calabria & $\begin{array}{l}\text { Punta Zambrone }\left(38^{\circ} 42^{\prime} 52,01^{\prime \prime}, 15^{\circ} 58^{\prime}\right. \\
\left.\quad 24,36^{\prime \prime}\right)\end{array}$ & 40 & $\begin{array}{l}\text { Sedimentary: sands, sandstones, limestones, } \\
\text { clays; Plutonic: monzonites, granites, } \\
\text { granodiorites; Metamorphic: gneisses }\end{array}$ \\
\hline Sicily & & $\begin{array}{l}\text { Milena - M.te Campanella } \\
\left(37^{\circ} 27^{\prime} 33,08^{\prime \prime},\right. \\
\left.13^{\circ} 43^{\prime} 20,16^{\prime \prime}\right)\end{array}$ & 80 & Sedimentary: clays, sands, evaporites, marls. \\
\hline \multirow[t]{2}{*}{ Sardinia } & & $\begin{array}{l}\text { Antigori }\left(39^{\circ} 05^{\prime} 43,76^{\prime \prime}, 09^{\circ}\right. \\
\left.00^{\prime} 17,35^{\prime \prime}\right)\end{array}$ & 98 & $\begin{array}{l}\text { Volcanic: andesites, basalts; Plutonic: granites; } \\
\text { Metamorphic: schists, phyllites, quartzites }\end{array}$ \\
\hline & & $\begin{array}{l}\text { Domu s'Orku (39॰ } 03^{\prime} 41,21^{\prime \prime} \text {, } \\
\left.09^{\circ} 01^{\prime} 25,41^{\prime \prime}\right)\end{array}$ & 99 & $\begin{array}{l}\text { Volcanic: andesites, trachytes; Plutonic: } \\
\text { granites; Metamorphic: schists, phyllites, } \\
\text { quartzites }\end{array}$ \\
\hline
\end{tabular}

Site numbers are as in Fig. 1

admittedly based on very small sample numbers, are evident from the descriptions below (Figs. 12 and 15).

\section{Northern Adriatic: Po Valley}

Mention has already been made of the group comprised of ICP-ES compositions of Aegean-type pottery from three sites in the Po Valley (Fig. 7). This group was problematic in two ways: it was numerically small, and there was a lack of uniformity in composition. Considering them now on an individual basis (Table 1), it is clear that among the Bovolone samples, Bov 4 has an anomalous composition and Bov 1-3 have inconsistent contents in individual elements such as $\mathrm{K}$ and $\mathrm{Mn}$; there are variable $\mathrm{Mn}$, Co and $\mathrm{Cr}$ contents among the
Lovara samples. But, on balance, sufficient coherence exists among the compositions to suggest that when compared with groups from other regions within Italy, they stand apart, as Fig. 7 indeed shows. Furthermore, they are significantly closer in their $\mathrm{Cr}$ and Co contents to most (modern) clays from the Po Valley analysed by Picon (2000) and to first millennium BC pottery from the Veneto region (Maritan 2004) (Jones et al. 2014, Database 5: Veneto) than to Mycenaean pottery from most parts of the Peloponnese, central Greece or central Crete (Jones et al. 2014, reference data in Database 2 and Appendix Table 12). The implication therefore is that the Po Valley group can be regarded as representing localised productions and thus its constituent members should be classed as IM.
Fig. 8 PC plot of the NAA compositions of IM at Coppa Nevigata (Adriatic), Torre Castelluccia, and Porto Perone (Eastern Ionian), Broglio di Trebisacce (Western Ionian) and Luni and Monte Rovello (Tyrrhenian). The main clusters in the dendrogram are superimposed. Individual compositions in Database 2 in Jones et al. 2014

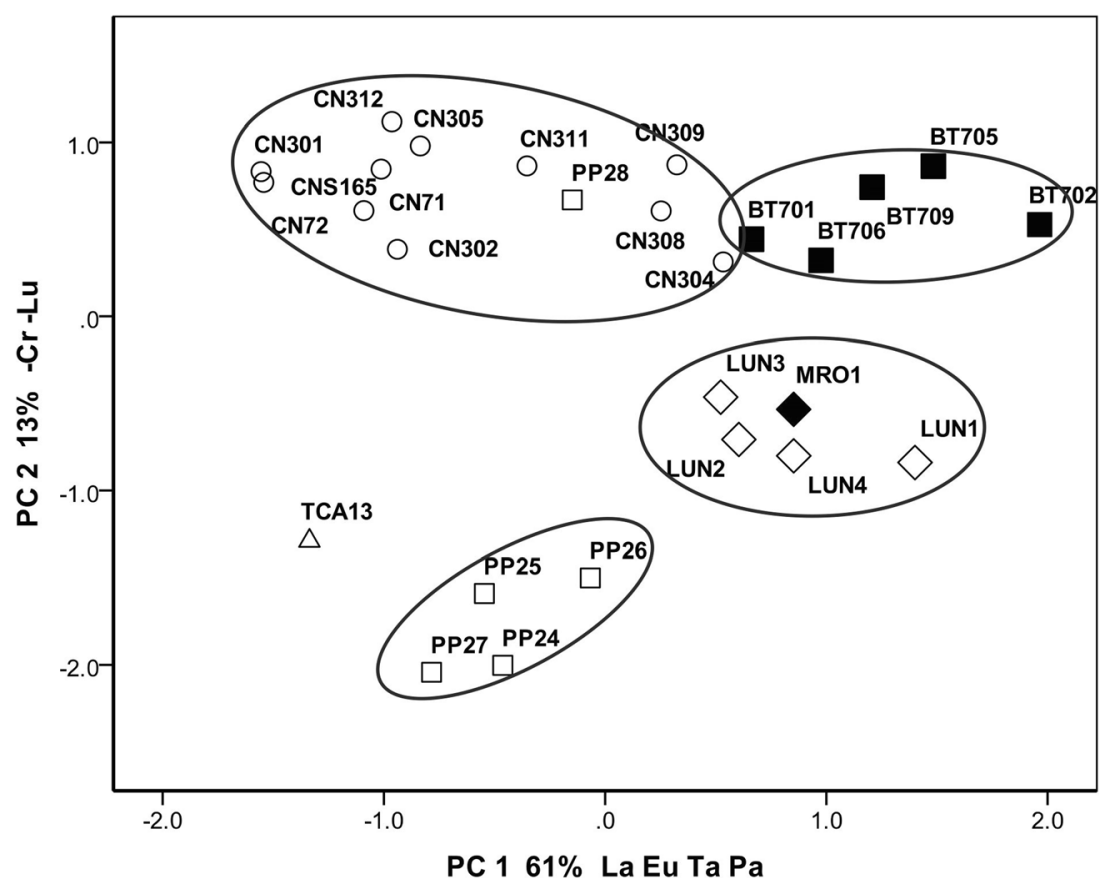


Fig. 9 PC plot of the ICP-ES compositions of IM at Coppa Nevigata (CN), Rocavecchia (RO), Torre Santa Sabina (TSS). I, II and III are the three main clusters from ALCA

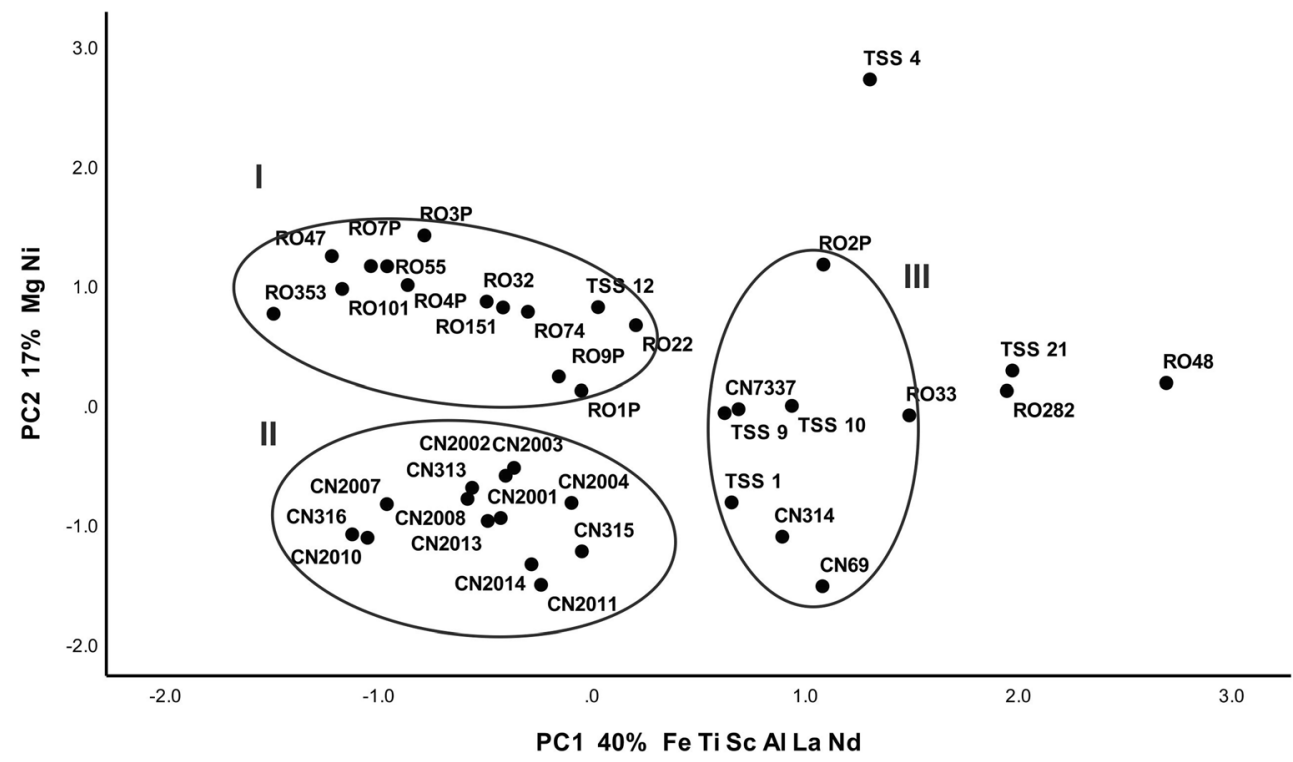

\section{Intra-site fine ware production}

This section considers the results of chemical and petrographic analysis of more than one class of Aegeantype pottery found at a given site. The issue is whether IM, Grey ware and the chronologically later Protogeometric and Geometric (PG/GE) wares, as described in the Introduction, were manufactured of the same clay, perhaps in the same general area. Such a comparison is possible at Coppa Nevigata, Rocavecchia and Broglio di Trebisacce.

\section{Coppa Nevigata}

Petrographic analysis of PG/GE ware shows the existence of three main compositions: calcareous with quartz, calcareous with fossils and micaceous (Table 5). Sample 1270 stands out having abundant iron oxides. All the samples are fine with only a fine fraction.

Chemically, one broad cluster (I in Fig. 11) accounts for the majority of IM and PG/GE examples and the one Grey ware; they are all likely to be products of related local clays. In petrographic terms, this cluster combines several identifiable fabrics, mainly micaceous and calcareous (Table 5, Fig. 12). The presence of iron oxides is reflected in the chemical compositions of the outliers 1270, 1172 (micaceous with oxides) and 2012, all of them having higher than average iron contents. The micaceous samples 508, 555 and 1267 appear in the lower part of cluster I, while the calcareous ones, such as 1263 and 1264, are closer to the IM samples. The relative lack of chemical uniformity in $\mathrm{PG} / \mathrm{GE}$ is reflected in the wide range of their scores on PC 1 in
Fig. 10 PC plot of ICP-ES compositions of IM at Coppa Nevigata and Tolentino. The groupings from ALCA are superimposed on the plot

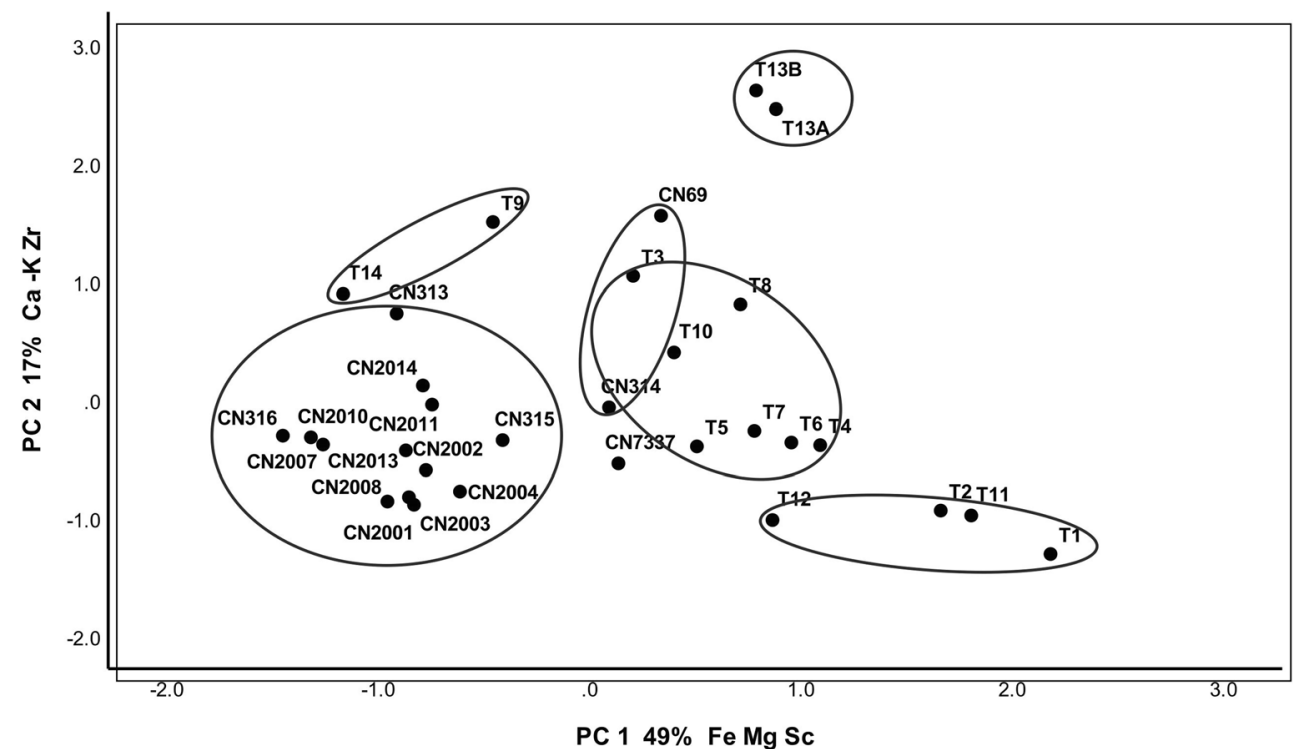




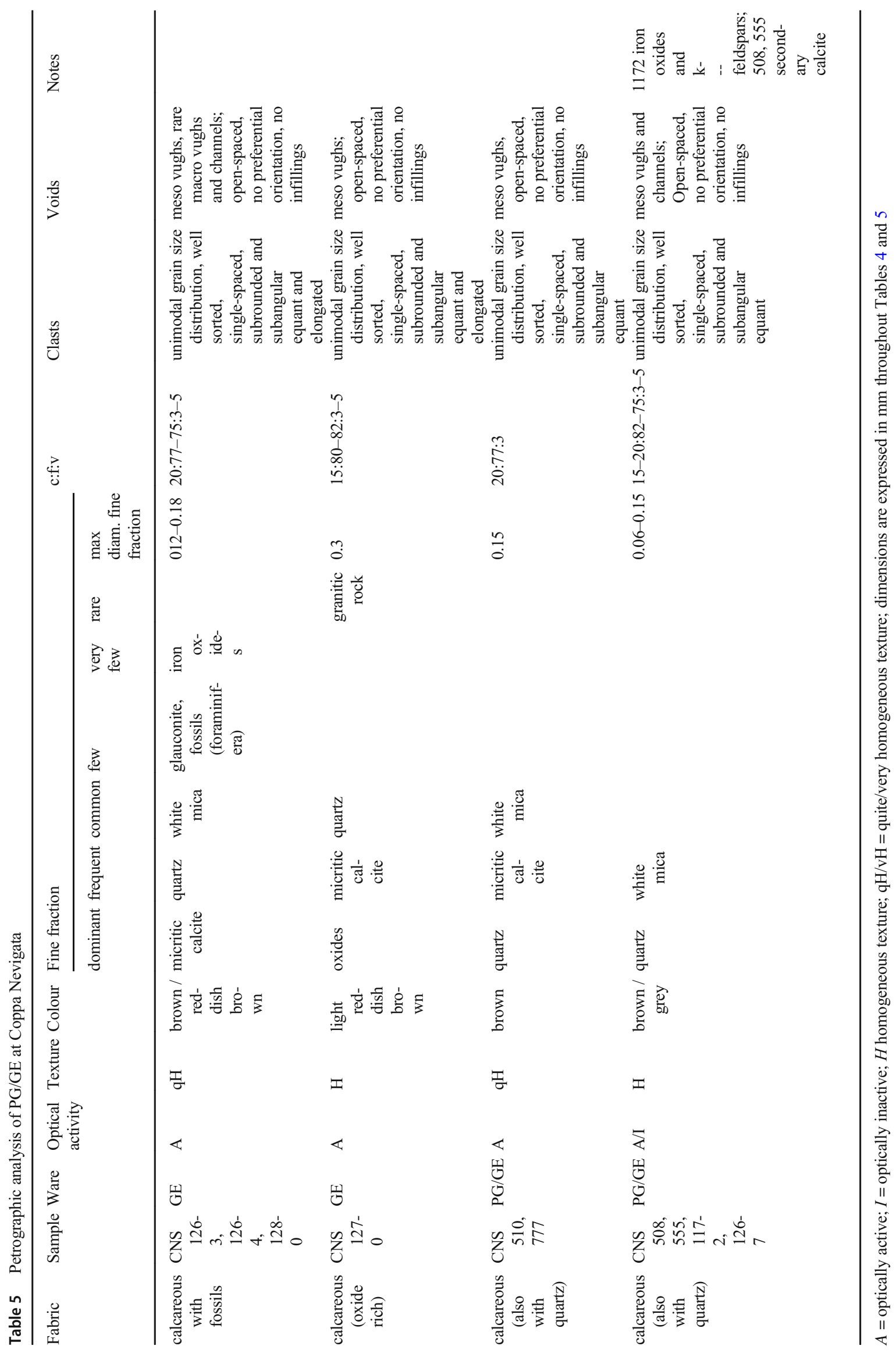


Fig. 11 PC plot of ICP-ES compositions of IM (black), Grey (diamond), PG/GE (black triangle) and Burnished (square) at Coppa Nevigata. The clusters from ALCA are superimposed on the PC plot

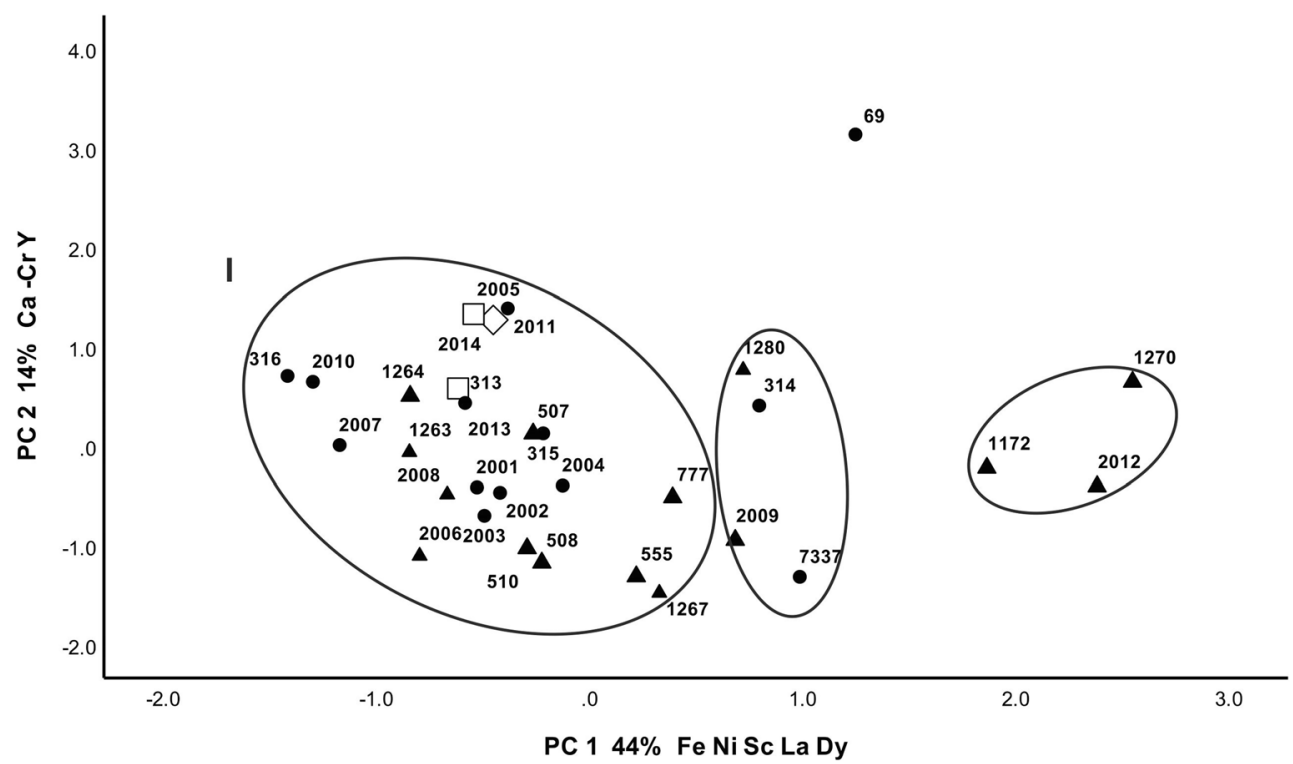

Fig. 11, but those examples of PG/GE and IM belonging to cluster I differ little from each other; the discrepancies in Mg and $\mathrm{K}$ are small (Table 2). IM 69 and 314 have already been shown to be atypical (Figs. 9 and 10); the former stands apart owing to its high $\mathrm{Ca}, \mathrm{Zr}$ and REE contents.

\section{Rocavecchia}

A different situation seems to be apparent here. Bivariate treatment of the data reveals higher levels of element correlation than at Coppa Navigata, for example, a significant Pearson $r^{2}$ correlation of -0.77 for Al-Ca as opposed to -0.14 at Coppa Navigata. That correlation feature is also apparent in the PC plot (Fig. 13) which reveals a single large cluster containing IM and the very small numbers of PG and Grey. There are proportionately more IM outliers than at Coppa Nevigata, and one Grey ware (27) stands well apart due to low $\mathrm{Mg}, \mathrm{K}$ and $\mathrm{Na}$. 9P is distinguished by low $\mathrm{Ca}$, high $\mathrm{K}, 48$ by high $\mathrm{Fe}, 2 \mathrm{P}$ by high $\mathrm{Cr}$ and 33 by high $\mathrm{Cr}$ and $\mathrm{Co}$.

The petrographic features, summarised by Cannavò (in Jones et al. 2014, 50-51) (here Fig. 12), are as follows:

IM 22-Fine texture, quartz, mica, iron oxides, carbonates and foraminifera microfossils. This sample is characterised by the presence of fossils in contrast to the other four samples from the same site which were chemically defined as imports from the Peloponnese.

GR 27, 28-The matrix is fine, light grey with homogeneous and isotropic texture. Abundant microfossils and carbonates are present; few quartz with sub-rounded monocrystals; and little quartz is present with sub-rounded monocrystals, fresh flakes of muscovite, feldspars and iron oxides.
PG 5, 15, 16-The matrix is fine, light brown with homogeneous and isotropic texture. Quartz is present with sub-rounded/angular monocrystals, fresh flakes of muscovite, feldspars, iron oxides, pyroxene, fossils and carbonates. Few ARF are present, probably argillaceous rock fragments (ARF). PG 16 is characterised by the presence of fossils of millimetric size.

\section{Coppa Nevigata, Rocavecchia, Torre Santa Sabina and Scoglio del Tonno}

Figure 14 considers collectively the fine wares-IM, GR and PG/GE - at sites in present-day Apulia as a whole; the four main clusters, I to IV, from ALCA are placed on the PC plot. It is encouraging to find broad inter-site differentiation for all three wares. Rocavecchia and Coppa Nevigata IM separate from each other along PC 2, dominated by origin-sensitive elements, in keeping with their classification in Fig. 9. Most of the IM from both sites have negative scores on PC 1 along with Grey ware and PG/GE, cluster I for Rocavecchia and cluster II for Coppa Nevigata. On the other hand, IM and Grey ware at Scoglio del Tonno are somewhat separate, classifying mainly in cluster III. As observed in Fig. 9, the TSS samples are manifestly not uniform.

As for the members of the poorly defined cluster IV, CN 1172,1270 and 2012 remain separate from the main $\mathrm{CN}$ group as previously observed in Fig. 11, while 1280, an outlier in Fig. 11, is now better placed in the local cluster I. Little can be said at present about IM 33, 48 and 282 at Rocavecchia in cluster IV. 
Fig. 12 Photomicrographs in XPL of thin sections of PG/GE from Coppa Nevigata and IM, GR and PG from Rocavecchia. Horizontal axis: $5.5 \mathrm{~mm}$

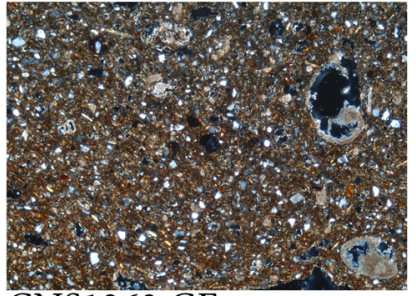

CNS1263 GE

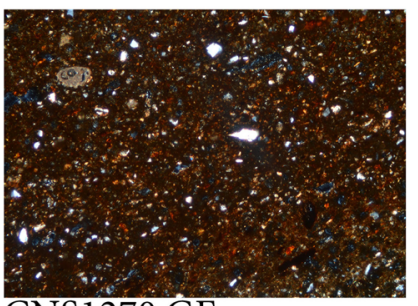

CNS1270 GE

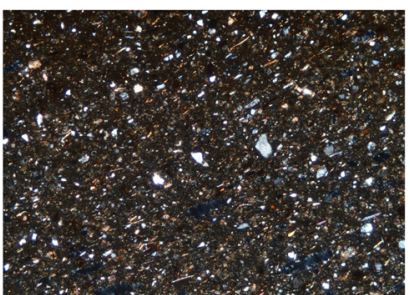

CNS508 GE

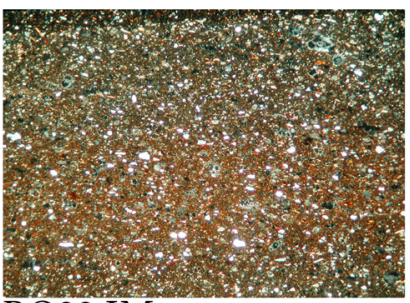

RO22 IM

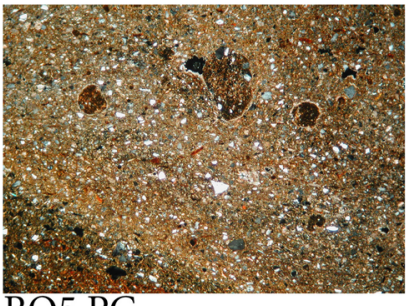

RO5 PG

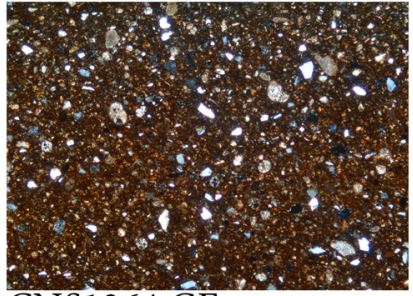

CNS1264 GE

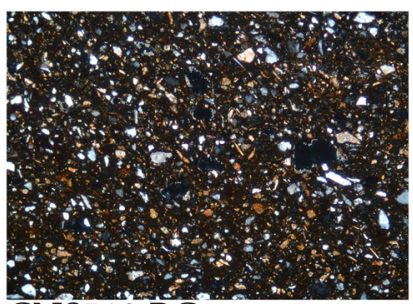

CNS510 PG

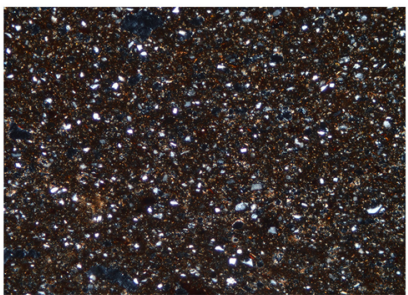

CNS555 GE

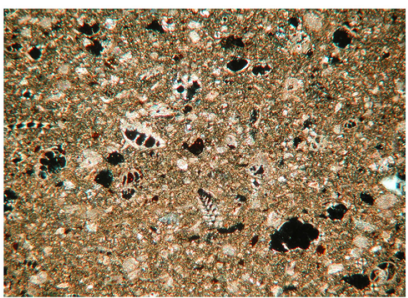

RO27 GR

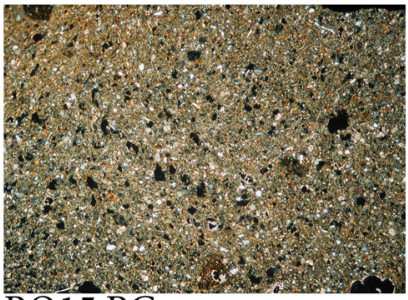

RO15 PG

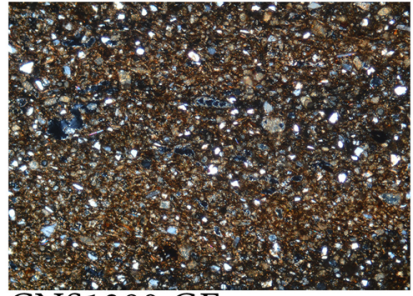

CNS1280 GE

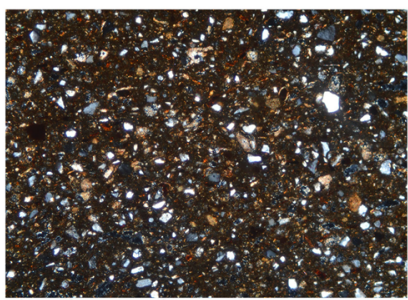

CNS777 GE

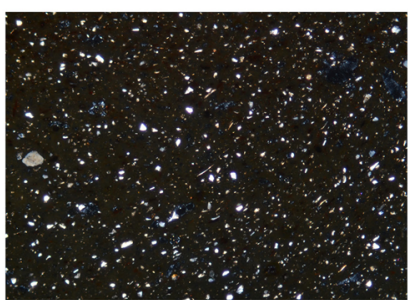

CNS1172 PG/GE

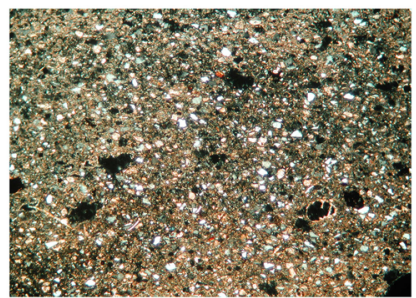

RO28 GR

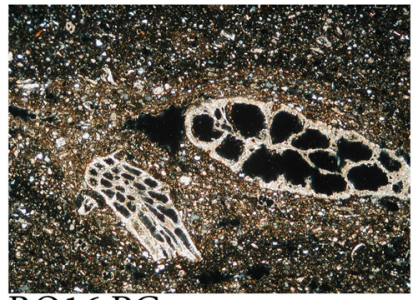

RO16 PG

\section{Broglio di Trebisacce}

The first phase of chemical work (using AAS) indicated that IM, GR and PG/GE had similar compositions (Jones et al. 2014, Fig. 4.15). The results of the corresponding NA analyses (Jones et al. 2014, Fig. 4.17), viewed in a Co-Cr plot, differed: in addition to a main group comprising IM, GR and $\mathrm{PG} / \mathrm{GE}$, there was a smaller one, also comprising mainly $\mathrm{PG}$, with higher $\mathrm{Cr}$ and $\mathrm{Co}$ contents which was interpreted as an atypical production in the Plain. And further scrutiny of the whole NAA data set has indicated the presence of a further group of five - all GE/late GE vessels - with low $\mathrm{K}, \mathrm{Rb}$ and 
Fig. 13 PC plot of ICP-ES compositions of IM (full circle), GR (square) and PG (black triangle) at Rocavecchia

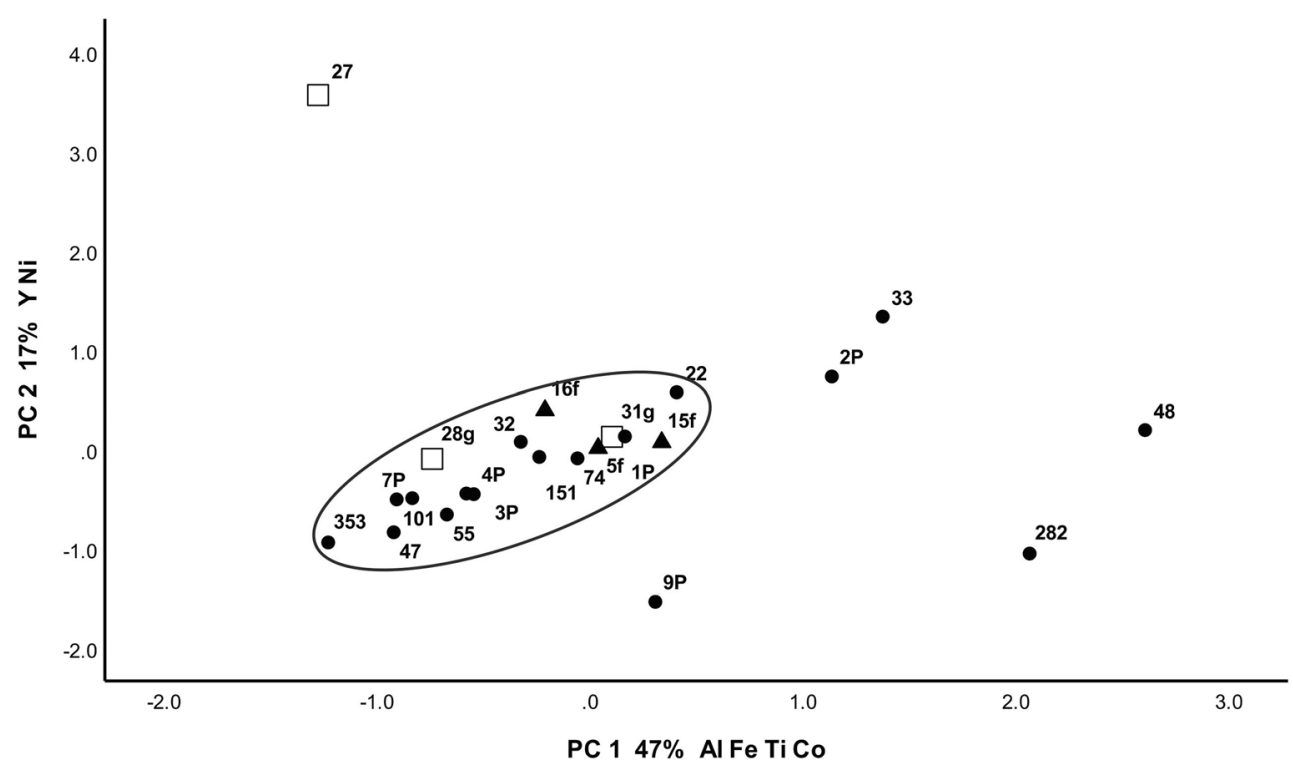

Cs: BT 801 (incised), 803 (handle), 811 and 833 (necked jars) and 855 . Two points can be emphasised thus far: first, there appears to be significant variation within PG/GE. Second, K, $\mathrm{Rb}$ and $\mathrm{Cs}$ were exactly those elements that were found in earlier work on the coarser-textured wares, dolia and impasto, found at different findspots within the Plain of Sybaris as a whole, to be indicative of a broad production location (Jones et al. 2014, 97f): high concentrations were associated with the clay and more particularly the (siltstone and shale) temper present in these wares in the north of the Plain. By contrast, $\mathrm{Na}$ was in higher concentration in the same wares in the south of the Plain, correlating apparently with greater plagioclase feldspar present in the temper (Levi 1999, Figs. 59-60; Jones et al. 2014, 192, 196).

Early petrographic work at Broglio by Lazzarini and Mariottini (in Jones et al. 1994) examined Mycenaean and
IM (17 samples), Grey (15) and PG/GE (3) (Jones et al. 2014, Table 4.7a and DB4). Having shown some variability in composition, this data set confirmed the existence of local/ regional products, for example, evidenced by the presence of siltstone in one IM sample (A72). IM production at Broglio itself was also strongly supported by the presence of an overfired IM amphora, the so-called 'green vase' (A65).

The more recent but previously unpublished petrographic (P) and ICP-ES data for IM at Broglio can now be introduced (P 4 samples; ICP-ES 7), GR (P 22; ICP-ES 30), PG (P?; ICPES 15), GE (P?; ICP-ES 21), PG/GE (P?; ICP-ES 8) and (possible) Archaic (P and ICP-ES 1) (see also Table 1 for each sample's chronological classification). The petrographic results for IM (Table 6 and Fig. 15) indicate a micaceous fabric with quartz and rare occurrence of granitic rocks in two samples; chemically the IM forms a reasonably coherent group
Fig. 14 PC plot of ICP-ES compositions of IM (open circle), Grey (diamond), PG/GE (black triangle) at Rocavecchia (RO), Coppa Nevigata (no prefix), Scoglio del Tonno (ST) and Torre Santa Sabina (TSS)

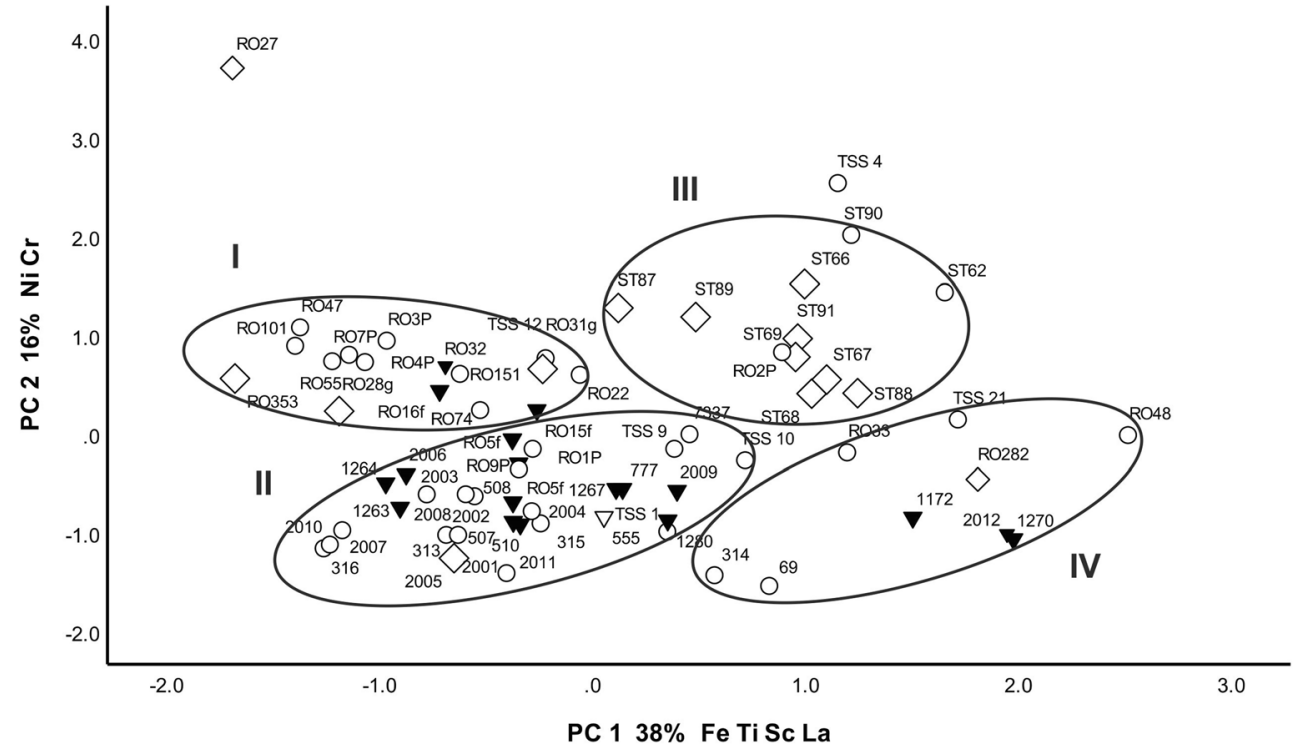


(Table 4). By contrast, GR ware is petrographically significantly more variable than IM (Table 7 and Fig. 15) and PG and GE yet more so (Table 8 and Fig. 15). An important feature of both PG, GE and GR wares is the presence of coarse components in some fabrics. There is a lack of any chronological difference between the fabrics in which PG and GE appear.

A global view of the chemical relationship between the wares using PCA indicated a main cluster comprising all three wares but with a notable presence of GR, PG and GE samples outside that main cluster: GR 609, 613, 616, 623, 631, 634, 635 and 636; PG 816 and 822; PG/GE 870; GE 871 and 875; and (possible) Archaic 872.

Treating the Grey ware on its own by PCA classifies this ware into several apparent groups, some of them according to Na content (on PC 2 (15\% of total variation)) which has been identified, as already mentioned, as one of the diagnostic elements separating coarser-textured products (dolia and impasto) of the northern from the southern part of the Plain. For the Grey wares, PCA suggested the presence of a small group including $609,613,616$ and 623 with high scores on PC 1 (57\% of total variation) separating from the diffuse central cluster. This group retained its identity when PCA was repeated using element to $\mathrm{Sc}$ ratios. But the way the major/minor elements, $\mathrm{Al}, \mathrm{Fe}$ and $\mathrm{Ti}$, accompanied by others, mainly trace elements loaded PC 1 pointed to a high level of inter-element correlation; this prompted us to resort to a simpler means of examining the structure within the data.

The Al-Ca plot (Fig. 16a) reveals a broad central cluster with a separate high $\mathrm{Al}$ content group whose members (609, $613,616,623,631,634)$ have the following petrographic features: iron oxides (616 and 634), quartz and grog (609 and 623) and micaceous (631). The calcareous Grey ware 625 is joined by chemically more calcareous 624 with low Al content and 635, 636 (both with quartz and mica) and maybe 633 (grog and siltstone and micaceous). The central cluster seems to be characterised by calcareous fabrics with fine (white) mica, quartz and quartz and mica. The plot of the origin-sensitive elements, $\mathrm{Cr}$ and $\mathrm{Ni}$, shows the expected high level of correlation but with many of the samples in the Al-Ca plot setting apart again from the main cluster (Fig. 16b): 623 and 634; 631; and 616, 609 and 613 (all, except 616, belonging to non-calcareous fabrics). On chemical grounds alone, these outliers could be regarded as products of a different clay from that/those of the main cluster, with the corresponding petrographic data laying greater emphasis on the presence of inclusions of different natures and quantities. In any case, the size of the main cluster is surely a reflection of two factors: textural variation as observed in the petrographic data (Fig. 15), giving rise to a dilution effect, and natural variation in the two elements in spatially similar clay deposits.

Grey ware displays great variability in the colour of both the surface and the fabric. The majority can be classified in the 
Fig. 15 Photomicrographs in XPL of thin sections of IM, GR and $\mathrm{PG} / \mathrm{GE}$ from Broglio di

Trebisacce. Horizontal axis: $5.5 \mathrm{~mm}$
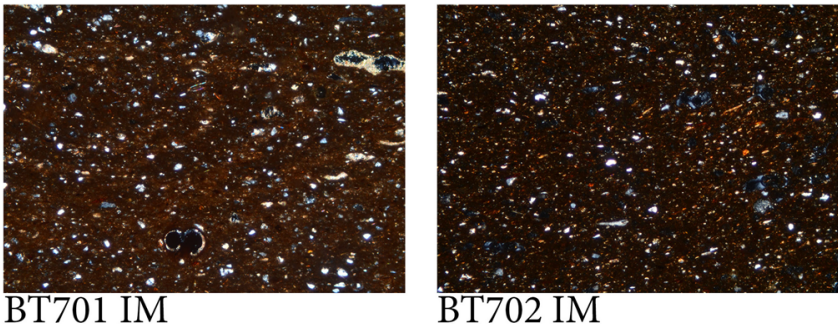

BT702 IM

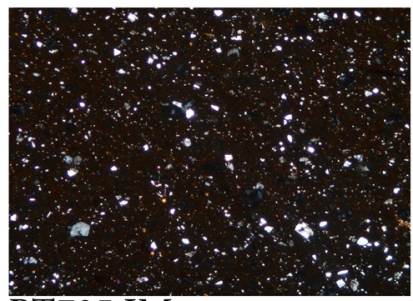

BT705 IM

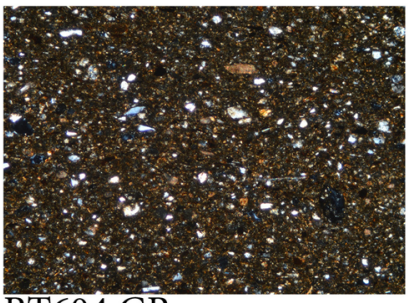

BT604 GR

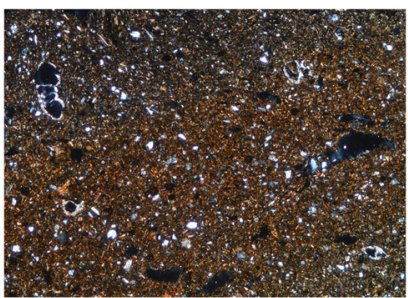

BT602 GR

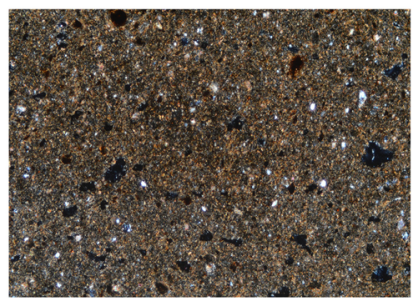

BT606 GR

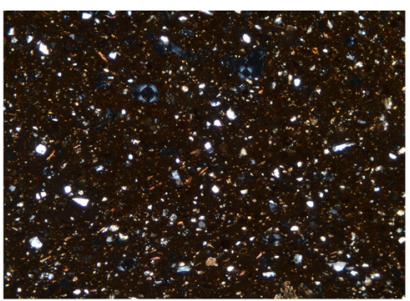

BT605 GR

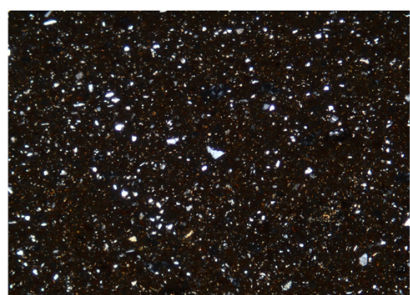

BT630 GR

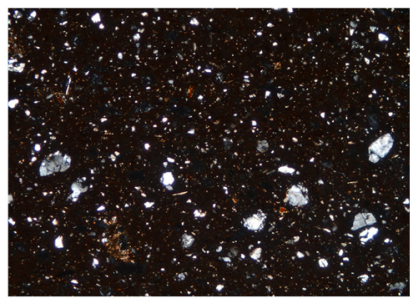

BT802 GE

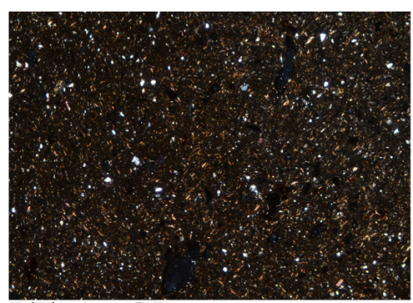

BT631 GR

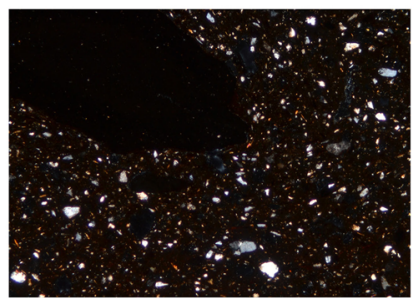

BT835 GE

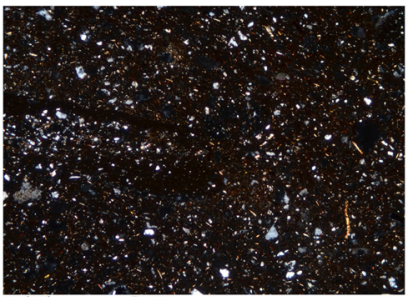

BT858 GE

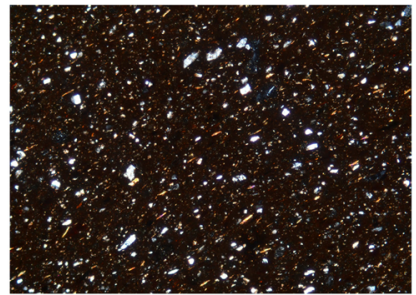

BT801 GÉ blue range (from 5BG to $5 \mathrm{~PB}$ Hue on the Munsell scale) with different degrees of lightness and saturation. One group is more yellowish and usually light. Investigating the possibility of specific choices made by the potters in order to obtain different colours revealed no evident correlation between colour variation and composition of the ceramic paste. In particular, those specimens with a higher $\mathrm{CaO}$ content and, according to petrography, calcareous components did not have lighter colours.

Multivariate classification by PCA of the results for PG, GE and PG/GE again shows a distinct central cluster with several scattered outliers (or loners): 815 (quartz); 816, 870 


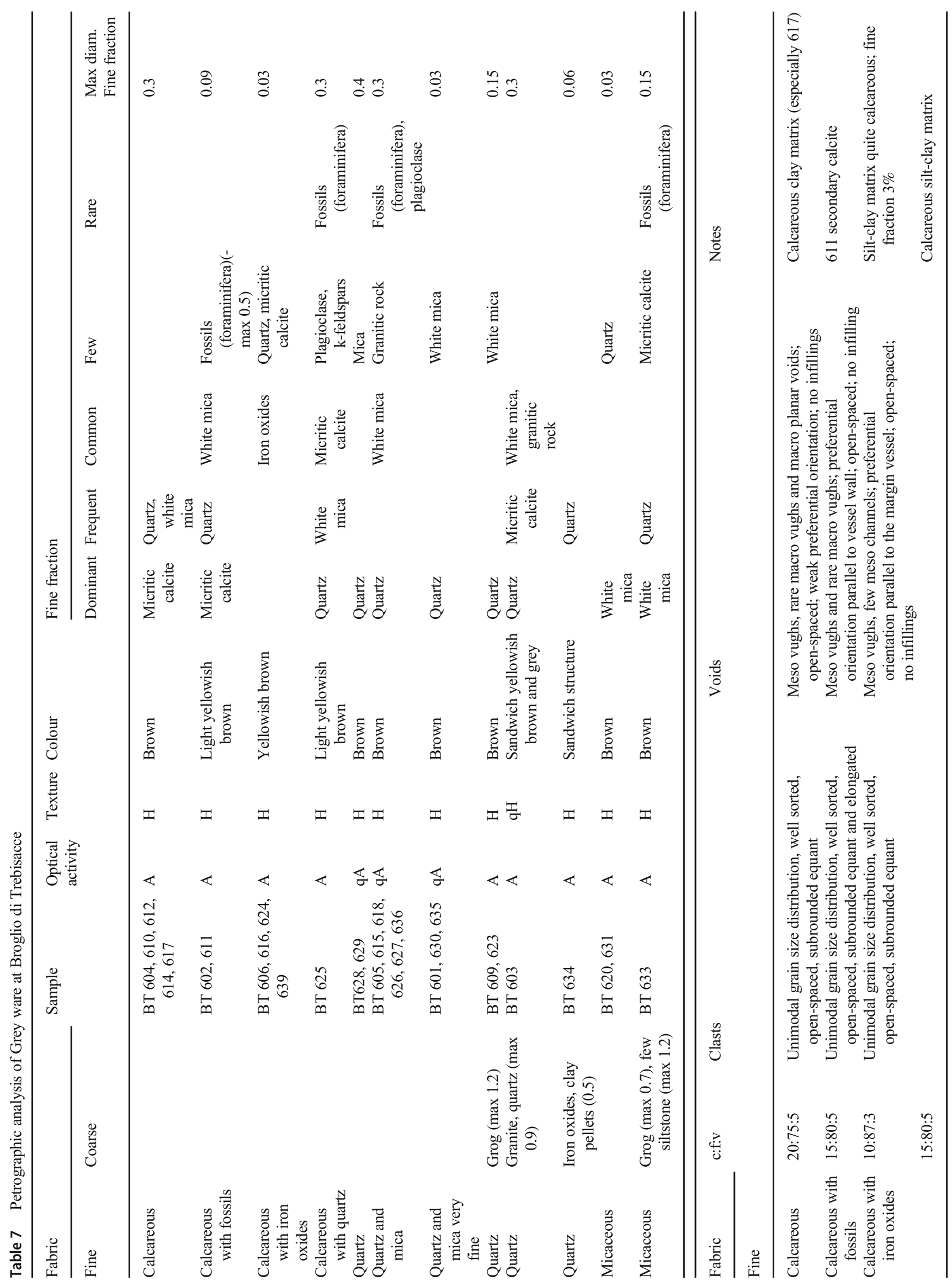




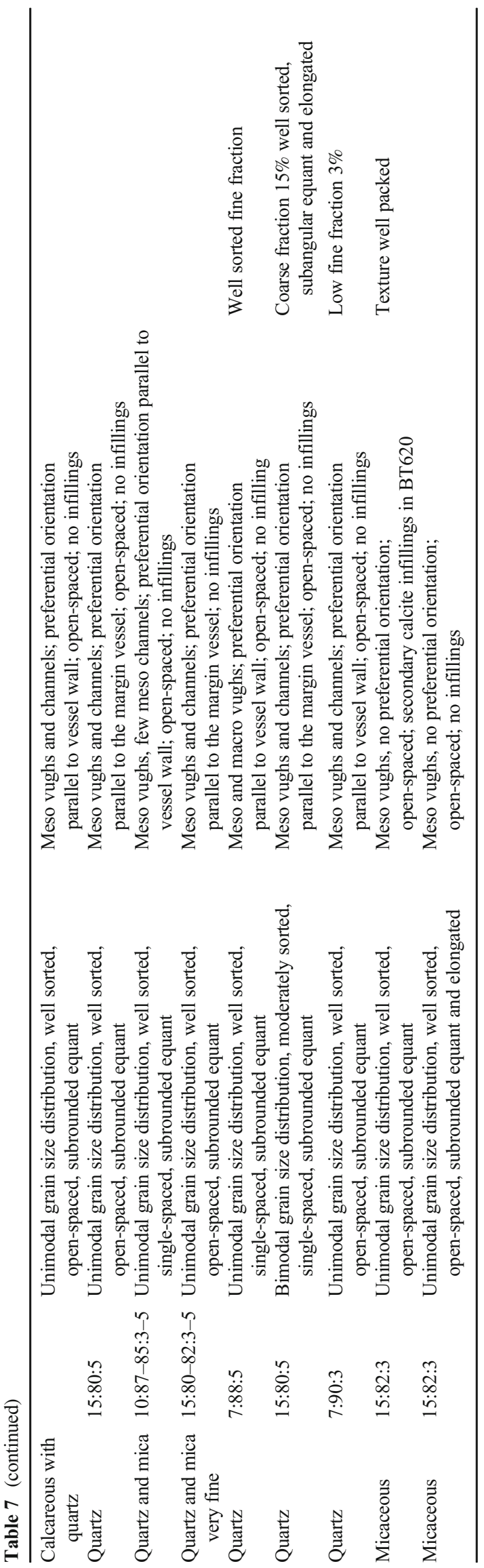

and 871 (quartz and grog); 801 and 872 (micaceous); 885 and 878 (not analysed petrographically) and, at the opposite side of the main cluster, 822 (calcareous). These are also evident in the bivariate plots (Fig. 17a, b); PG and GE share with GR the features of high $\mathrm{Cr}-\mathrm{Ni}$ correlation, unusually wide ranges in these two elements and a small high Al group (comprising one example each of PG, GE and (possible) Archaic) in the Al-Ca plot. It is evident that chemically similar clays were used for both PG and GE.

In summary, the picture emerges that much Grey ware was produced alongside IM at a central location which is most likely to be Broglio itself. But at the same time, the petrographic results confirm an underlying variability in Grey ware and, to a greater extent, in PG and GE wares, a finding that is helped by having good sample numbers; the wares share some of the same fabrics, e.g. calcareous and micaceous, which is no cause for surprise, while PG appears in fabrics not observed in either IM or Grey (or anything else). The PG and GE fabrics with clasts in which each member is in a sense unique hint at individual sui generis production, and similar remarks apply to Grey ware. A model of dispersed production of Grey, PG and GE within the Plain seems reasonable to propose, and this is supported by the corresponding chemical data: first, the NAA-defined disparate group of PG/GE samples whose low contents of mineralogically sensitive elements, $\mathrm{Rb}$, Cs and $\mathrm{K}$, point to a source in the southern part of the Plain (see above in this section); second, ICP-ES compositions of a small group of PG and GE (BT 827 and 847 (micaceous), 853 (calcareous) and 874) with significantly lower $\mathrm{K}$ and $\mathrm{Rb}$ contents than the remainder (Table 1). While the main chemical group encompasses examples of the various fabrics, the high Al group in Fig. 16a does not appear to have a single petrographic equivalent. Searching for comparanda of that high Al group in the combined AASICP data set in the Plain of Sybaris has not yet been successful.

\section{Discussion}

The study of the style and technique of IM has emphasised the variability of this ceramic class which in turn is informative about the identity of the potters concerned (Bettelli in Jones et al. 2014, 455). At Rocavecchia, for example, it can be argued that Aegean potters were present, whereas at Broglio where a Cretan influence is discernible, there is maybe a combination of Aegean tradition and local innovation. And at Antigori on Sardinia (Table 3), the lack of familiarity with either the wheel or painting technique is more consistent with a local potter who had limited exposure to the Aegean tradition. Traits are observed individually in several large IM assemblages leading to the idea of site-specific styles: Broglio di Trebisacce, Termitito and Coppa Nevigata styles, for example (Bettelli in Jones et al. 2014, 456). 


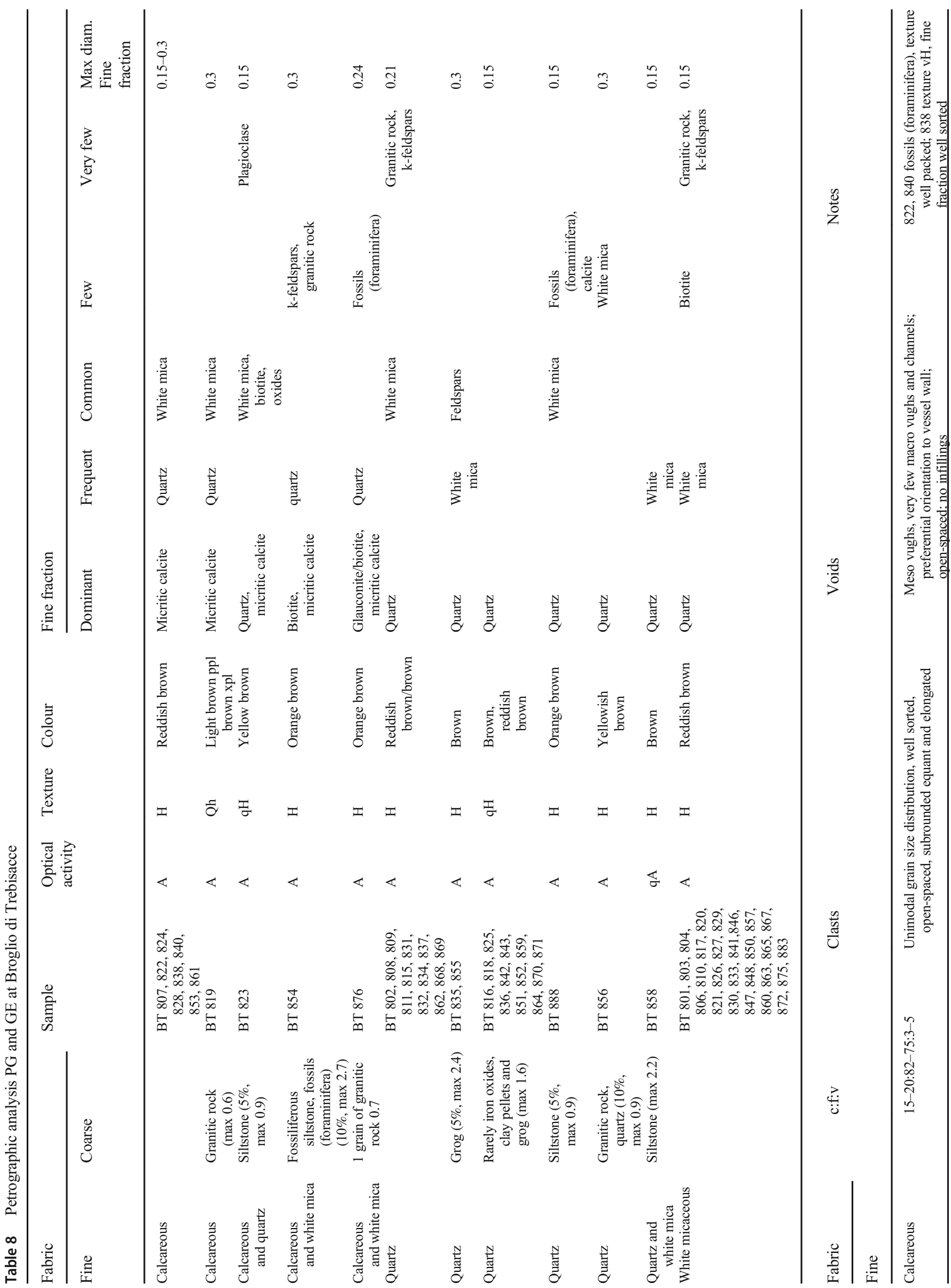



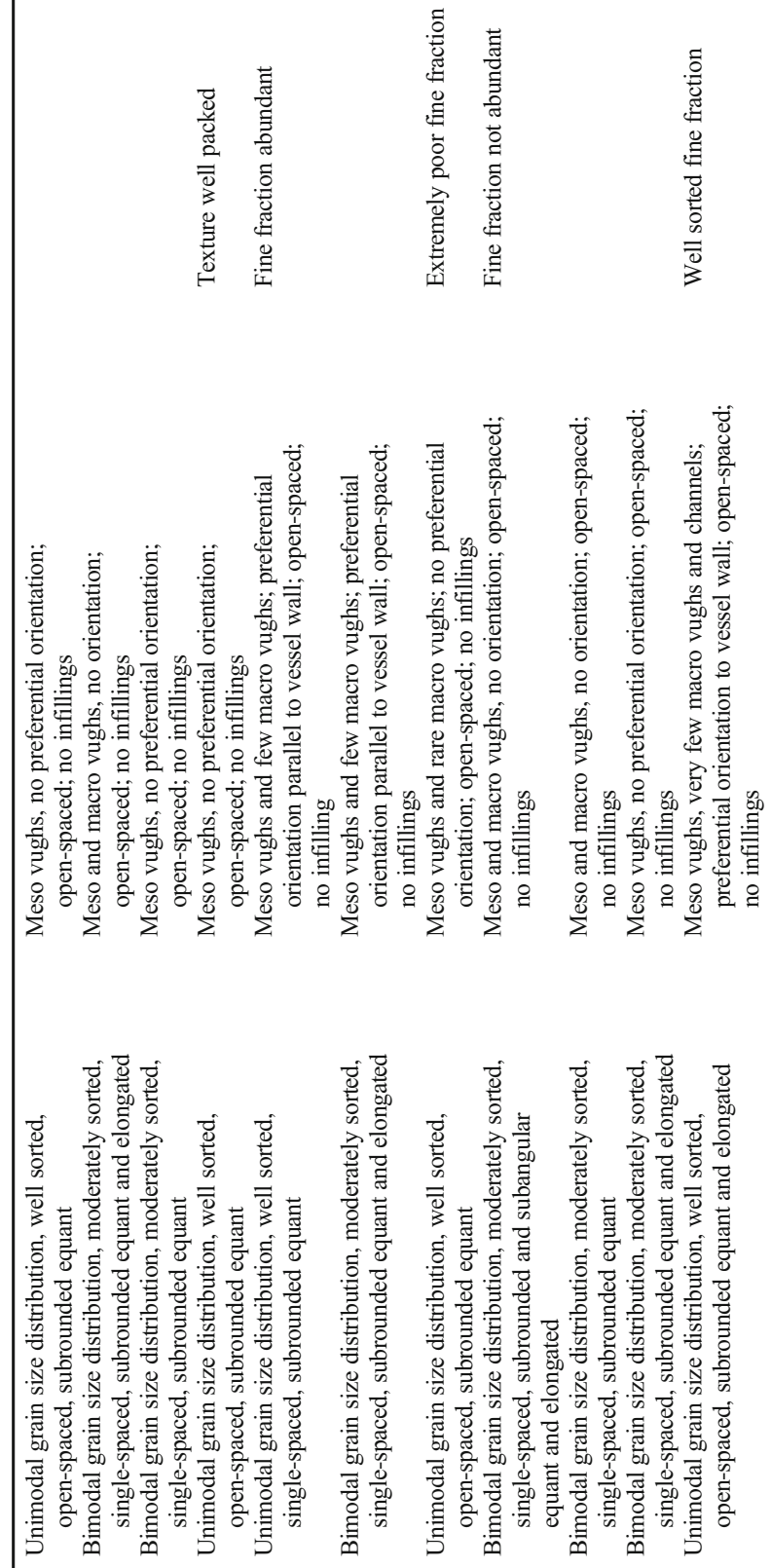

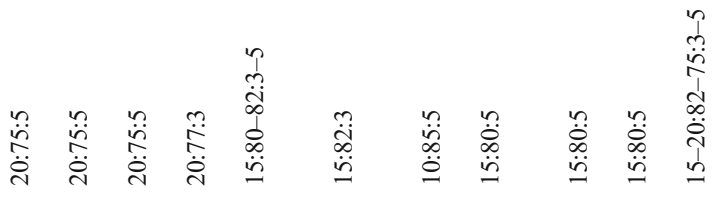

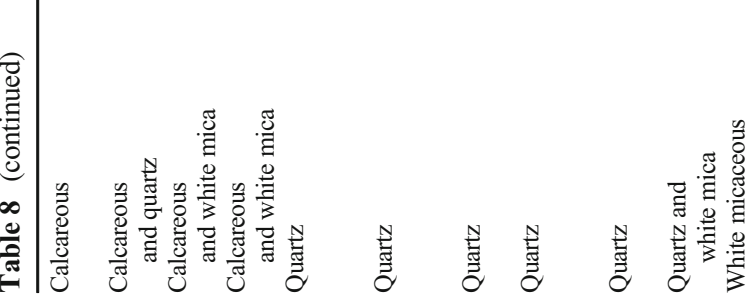


a

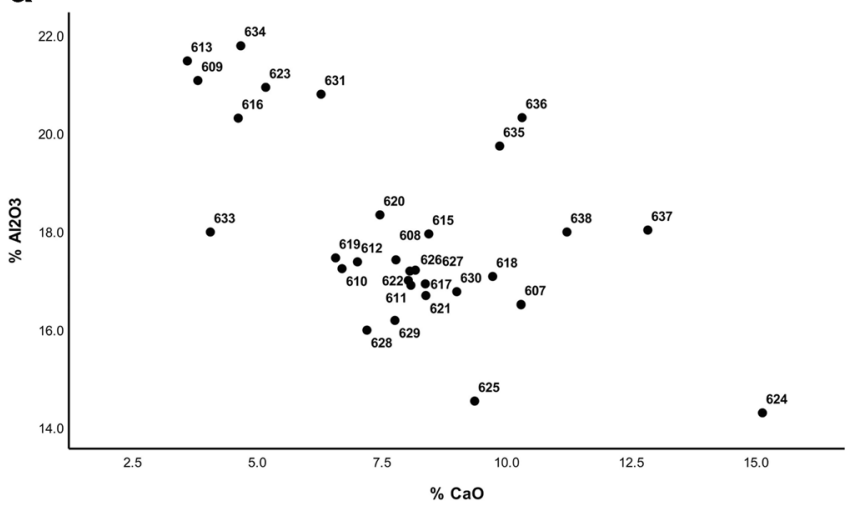

b

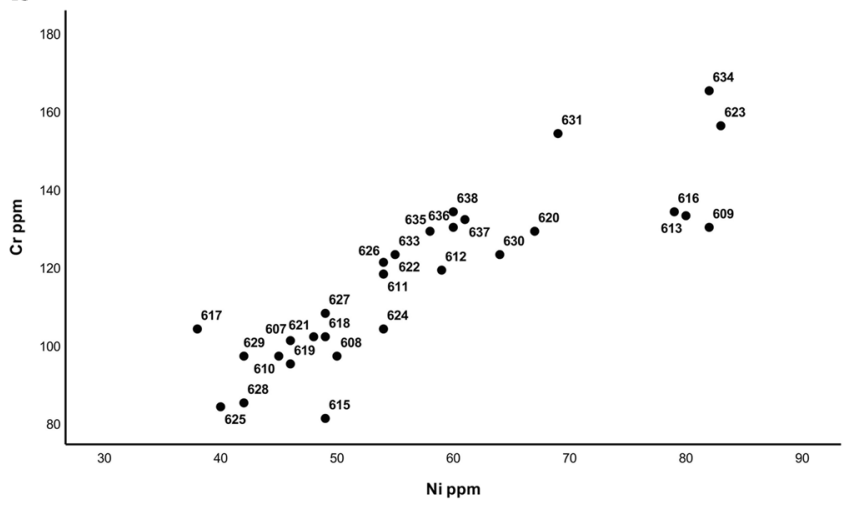

Fig. 16 a Al-Ca and b Cr-Ni plots for Grey ware at Broglio di Trebisacce. ICP-ES data

The most satisfactory outcome of the present investigation is the independent support it gives to these views based on style and technique. The support provided by interpretation of the chemical data is strong if uneven: wherever IM has been found in Italy and on Sardinia, it is most likely it was made locally or nearby. There is no clear evidence for the movement of IM from one region to another; the potter, whoever he was, rather than the product itself moved to the point of demand/ consumption, establishing overtime workshops in which also local apprentices might operate. Furthermore, there seems to be a sense that Aegean potters, having reached a base, operated from that base, perhaps moving between different settlements in a given region, such as Apulia, according to demand but not moving further afield. In other words, the Aegean potter who arrived at Rocavecchia had an ample market for his products there and would not have found it necessary to move on to fresher fields westwards or northwards on the mainland of Italy. That sense is one of the considerable directionality: Aegean potters may have targeted their individual destinations within Italy.

Just as there is variability in style and technique in IM, there is corresponding variability in the composition not only of the fabric but also the decoration. One explanation for this striking observation is that they reflect the additive contributions of several factors: first, the chronological duration of IM production - more than three centuries. Second, during that duration, the output is not of standardised pottery - a hallmark of decorated Mycenaean pottery - but rather of an evolving tradition of manufacture, one that starts with the original Aegean potter who established his workshop in Italy having found suitable raw materials but is still adapting to new conditions. According to our model, that tradition then develops as the process of technology transfer to the indigenous potter(s) progresses. Over the course of time, the trained indigenous potter(s) could also be operating in an independent capacity. In that scenario, it would then be surprising if a lack of uniformity in clay composition were not observed among examples of IM at a given site. The outliers and minor groups of IM, as at Coppa Nevigata (Figs. 9 and 10), therefore need not be problematic: they may represent the products of clays that were not the favoured ones, perhaps exploited early on but later went out of use. Alternatively, they were the products of established but minor local workshops. Unfortunately, it is unlikely that these outliers and minor groups have any meaning stylistically or chronologically to allow us to test these ideas. On the other hand, these ideas are consistent with the observed variability in decoration. The results of our technological enquiry repeatedly demonstrated a

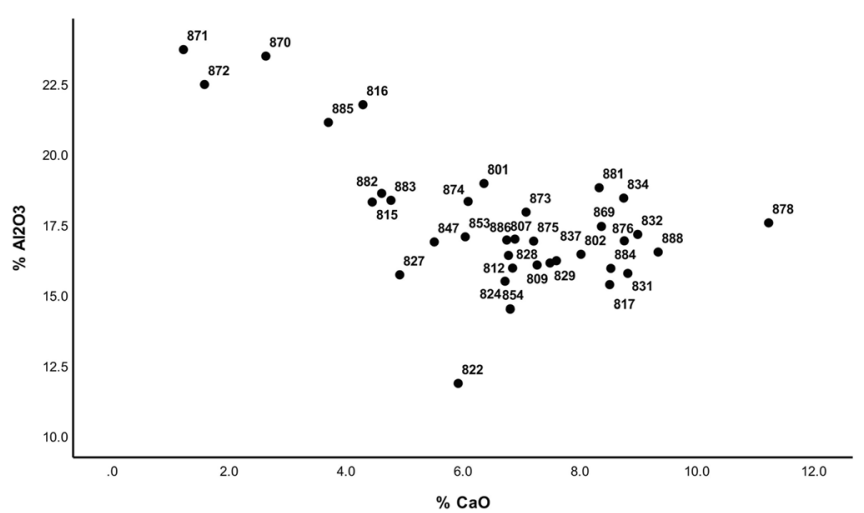

b

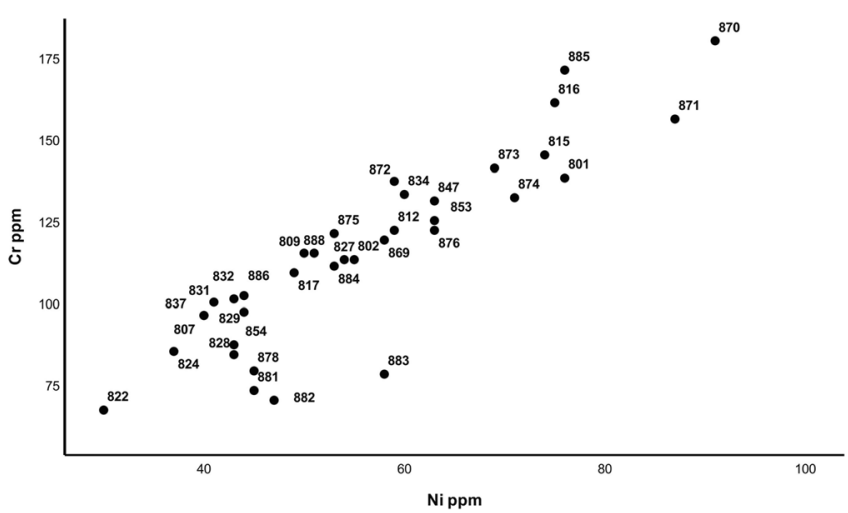

Fig. 17 a Al-Ca and $\mathbf{b}$ Cr-Ni plots for Broglio PG/GE ware at Broglio di Trebisacce. ICP-ES data 
the relative lack of uniformity: the technological tradition of IM production - use of iron-rich clay-based materials for the painted decoration and double chamber kiln firing that formed part of the experimental work presented by Vanzetti et al. (2014) - followed a fairly constant path but its execution did not. Considering the examples from Coppa Nevigata, Rocavecchia and Broglio di Trebisacce, the paint layer varies in thickness and quality (Jones et al. 2014, Table 5.4); and a consistent, even firing was not maintained as the firing temperature estimates indicate (Jones et al. 2014, Table 5.2).

As far as Grey ware is concerned, considering the plurality of production centres that it shares with IM, it shows a different behaviour in terms of style. In fact, the several production centres in south-eastern Italy - that is, the area in which Grey ware spread from the time of the Middle until the beginning of the Final Bronze Age-shared not only the technological package transmitted from the Aegean but also most of the shapes. Thus, a greater uniformity in terms of style is attested, different from that of IM, as noted above. This phenomenon could be attributed to potters who manufactured mostly Grey ware using local impasto shapes, and these were largely shared by local communities at that time. This observation leads us to consider two possible scenarios regarding the two Aegean-inspired wares: either they were produced in different workshops or mainly local potters - trained in the use of Aegean technologies - were involved in producing Grey ware even in the same workshops in which IM was made. That the consumer, who used and perceived the two wares differently, may have played a role in the contrasting stylistic choices is not denied in either scenario. In this way, IM and Grey wares seem to take on a different social "weight" within and among local communities, with only the former performing specific social functions as discussed below. The characteristic of shared shapes and decorative motifs is also present in PG, a ware made by local potters in a very local style, reproducing impasto shapes and decoration very common in the Italian Final Bronze Age.

The differing behaviours adopted by native groups in the production and use of IM occurred at contemporary sites sharing many characteristics such as settlement organisation, architecture and, in many cases, material culture in terms of bronzework and local pottery style (Castagna 2002, 2004; Iacono 2015; Bettelli in press-b). But these behaviours may also be justified by the constant construction and negotiation of identities taking place among local communities or perhaps only in certain sectors of them. This is important as it occurred during a period in which local communities had come into possession of exotic artefacts, technologies and socio-ritual practices. Taken together, these features may have had a restricted circulation; certainly, no community in the regions under consideration could boast, in this late phase of the Bronze Age, exclusive knowledge and use.
The local manifestation of these material symbols of social distinction could allow greater control in terms of their production, circulation and above all consumption, without having to depend on the fluctuation in supply that was always a risk when relying exclusively on overseas trade. The local production of Aegean-type pottery with the development of specific styles, other than in terms of social identity in its various forms, could also be explained by the desire to avoid making use of local exchange networks for the possession of such socially crucial assets, networks that could have been managed by rival elite groups (Bettelli in press-b; Bettelli and Levi in press).

The weakening and subsequent interruption of the links with the Aegean towards the end of the second millennium $\mathrm{BC}$, whose causes are mainly sought in the historical processes and phenomena that took place in Greece, did not promote further circulation of formal and decorative styles prevalent in the Aegean. Instead, it favoured the development of a ceramic style - PG - which had already been formed in general terms (see, for instance, Ausonian I (Recent Bronze Age) at Lipari (Bernabò Brea and Cavalier 1980)), characterised by the presence, both in form and decoration, of various types of native element (Yntema 2000, 320; Peroni 1994: 857-860; Vanzetti 2000; Bettelli 2008; Bettelli et al. 2018). The consumers of this tableware, probably members of the elite, no longer emphasised their social distinction with the use of 'exotic' ceramics, as was the case with IM. Instead, the shape and decoration of PG vessels aligned themselves to a local visual repertory, which additionally and above all included impasto ware (Yntema 2000, 320; Peroni 1994, 857-860). Moreover, unlike IM, PG productions, which can scarcely be identified in terms of regional style, have a greater stylistic homogeneity over large distances (Yntema 2000, 320; Vanzetti 2000). The analyses indicate multiple production centres, and so it seems that this stylistic consistency should be attributed to a broader sharing of models, rather than to the circulation of products. During the FBA, unlike previous periods, fine, painted, specialised ceramics may assume a new social function. This was more strongly subject to the expression of cultural identity and probably involving broad areas as well as expressing different sets of values (Peroni 1994, 860) from that of social representation alone. The latter had earlier manifested itself through the production and consumption of technologically and stylistically sophisticated and exotic pottery, IM (Bettelli et al. 2018). In this way, PG seems to inherit and develop, albeit in a different historical scenario, the social functions possibly carried out by Grey ware starting from an earlier period.

Brief comment is necessary on the programme of analysis at Punta Zambrone, mentioned above, by Jung et al. (2015) who found that four samples $(9 \%$ of the total analysed from that site) matched Mommsen's NAA SybB reference group which may represent production in the southern Plain of 
Sybaris. They claimed that this was possible evidence for the movement of IM pottery from the southern part of the Plain of Sybaris to Punta Zambrone. However, this view may not yet be secure; as the authors themselves admit, two of the sherds (Zamb 18, 20, 31 or 42 in Jung et al. 2015, Table 1) are not very diagnostic, and second two members of the SybB group that are linked to the southern part of the Plain (932A and 962 A) are dolia, not IM. Instead, IM features in Mommsen's SybA reference group which should be attributed to the northern part of the Plain as it includes five examples of IM from Broglio di Trebisacce (see Jones et al. 2014, 544, Table 10: Broglio 1, 4, 5, 69, 25). This issue is likely to be clarified in light of a forthcoming publication of new NAA data (H. Mommsen, pers. comm.)

Looking ahead, there is potential for further work in the Adriatic and Ionian zones as defined in Table 4. More sampling of IM in the Adriatic zone would be welcome to resolve the significance of the atypical IM at Rocavecchia (Fig. 13) as well as the variable IM from the smaller sites as observed at Torre Santa Sabina and Tolentino; was the IM at each of these sites made in more than one local workshop and/or was some of it made further afield within Apulia? Current archaeological and archaeometric work on IM and imported Aegean pottery to Sardinia is likely to shed new light on the status of the local production of pottery (c.f. Table 2), known from about ten sites on the island. Particularly promising are the finds from the new site of Selargius (Manunza 2016), east of Cagliari. The finds seem to have a certain degree of homogeneity in shape, decoration and finishing (L. Vagnetti and P.M. Day pers. comms.).

Acknowledgements The financial support from the Institute for Aegean Prehistory (INSTAP) and Preistoria Attuale is gratefully acknowledged. We are grateful to Lucia Vagnetti, the principal architect of the project from which the present work developed, for her general support and comment on an earlier draft of this paper. For permission to sample and for valuable discussion, we thank Alessandro Vanzetti at Broglio di Trebisacce, Alberto Cazzella and Giulia Recchia at Coppa Nevigata and Riccardo Guglielmino at Rocavecchia. We acknowledge the helpful comments of the reviewers.

Funding Institute for Aegean Prehistory (INSTAP) and Preistoria Attuale.

\section{Compliance with ethical standards}

Conflict of interest The authors declare that they have no conflicts of interest or competing interests.

Open Access This article is licensed under a Creative Commons Attribution 4.0 International License, which permits use, sharing, adaptation, distribution and reproduction in any medium or format, as long as you give appropriate credit to the original author(s) and the source, provide a link to the Creative Commons licence, and indicate if changes were made. The images or other third party material in this article are included in the article's Creative Commons licence, unless indicated otherwise in a credit line to the material. If material is not included in the article's
Creative Commons licence and your intended use is not permitted by statutory regulation or exceeds the permitted use, you will need to obtain permission directly from the copyright holder. To view a copy of this licence, visit http://creativecommons.org/licenses/by/4.0/.

\section{References}

Bernabò Brea L, Cavalier M (1980) Meligunis Lipara IV. L'acropoli di Lipari nella preistoria, Palermo

Bettelli M (2002) Italia meridionale e mondo miceneo. Ricerche su dinamiche di acculturazione e aspetti archeologici, con particolare riferimento ai versanti adriatico e ionico della penisola italiana, Grandi contesti e problemi della Protostoria italiana 5, Firenze, pp 233-249

Bettelli M (2008) Le ceramiche figuline dell'età del bronzo: importazioni, imitazioni e derivazioni locali. In: Bettelli M, De Faveri C, Osanna M (eds) Prima delle colonie. Organizzazione territoriale e produzioni ceramiche specializzate in Basilicata e in Calabria settentrionale ionica nella prima età del ferro, Venosa, pp 17-35

Bettelli M (2012) Due classi ceramiche di derivazione egea da Coppa Nevigata: la grigia tornita e la "burnished"a pittura bianca. In: Moscoloni M, Recchia G (eds) Cazzella A. Coppa Nevigata e l'area unida alla face del Candelaro durante l'età del bronzo, Foggia, pp $427-430$

Bettelli M (2019) I precedenti dell'età del Bronzo: il Campidoglio. Frammenti italo-micenei dagli scavi del Giardino Romano. In: Damiani I, Parisi Presicce C (eds) La Roma dei Re. Il racconto dell'archeologia, Gangemi Editore, Roma, pp 265-268

Bettelli M (in press-a) Specialisation, exchanges and socio-economic strategies of Italian Bronze Age elites: the case of Aegean-type pottery. In: Frangipane M, Poettinger M, Schefold B (eds) Ancient economies in comparative perspectives, Atti XV Convegno AISPE, (Roma, 23-25 novembre 2017)

Bettelli M (in press-b) From the Aegean to the Ionian Sea: pottery, technology and people in the Plain of Sybaris in the Late Bronze Age. In: Jung R (ed) 1200 B.C.E. a time of breakdown - a time of Progress in southern Italy and Greece, Proc. Internat. Congress (Rome, 16-18 April 2015)

Bettelli M, Levi ST (in press) Fifty shades of Italo-Mycenaean pottery: Frattesina and other Central Mediterranean nuances. In: Bellintani P (ed) Il Deltadel Po tra Europa e Mediterraneo nei secoli attorno al 1000 a.C., Atti del Convegno internazionale; Museo dei Grandi Fiumi di Rovigo 13-15 Aprile2018, Padusa LVI

Bettelli M, Cupitò M, Levi ST, Jones RE, Leonardi G (2015) Tempi e modi della connessione tra mondo egeo e area padano-veneta. Una riconsiderazione della problematica alla luce delle nuove ceramiche di tipo miceneo di Fondo Paviani (Legnago, Verona). In: Leonardi G, Tinè V (eds) Preistoria e Protostoria del Veneto. Studi di Preistoria e Protostoria 2, Firenze, pp 377-387

Bettelli M, Recchia G, Vagnetti L (2017) Ceramiche egee e di tipo egeo da Coppa Nevigata (FG): nuovi documenti. In: Radina F (ed) Preistoria e Protostoria della Puglia, Studi di Preistoria e Protostoria 4, Firenze, pp 473-479

Bettelli M, Borgna E, Levi ST (2018) Crisis years and pottery systems: an overview of the Italian Late Bronze Age. In: Caloi I, Langohr C (eds) Technology in crisis. Technological changes in ceramic production during periods of trouble. Louvain-la-Neuve, 18-19 February 2016, Aegis 16, Louvain-la-Neuve, pp 217-240

Broodbank C (2013) The making of the Middle Sea : a history of the Mediterranean from the beginning to the emergence of the classical world. Thames \& Hudson, London 
Buffa V (1994) I materiali del Bronzo finale e della prima età del ferro. In: Peroni R, Trucco F (eds) Enotri e Micenei nella Sibaritide I. Broglio di Trebisacce, Taranto, pp 455-569

Buxeda I, Garrigos J, Jones RE, Kilikoglou V, Levi S, Maniatis Y, Vagnetti L, Wardle KA, Andreou S (2003) Technology transfer on the periphery of the Mycenaean world: the case of Mycenaean pottery found in Central Macedonia and the Plain of Sybaris (Italy). Archaeometry 45(2):263-284

Cannavò V, Levi ST (2018) Atlas of ceramic fabrics 1. Italy: north-east, Adriatic, Ionian. In: Bronze age: impasto. Archaeopress, Oxford

Castagna MA (2002) La ceramica grigia tornita dalla "casa centrale" (campagne di scavo 1980-1982): una messa a punto. In: Bettelli M (ed) Italia meridionale e mondo miceneo. Ricerche su dinamiche di acculturazione e aspetti archeologici, con particolare riferimento ai versanti adriatico e ionico della penisola italiana, Grandi contesti e problemi della protostoria italiana, 5, Firenze, pp 233-249

Castagna MA (2004) I servizi da simposio in ceramica d'impasto e depurata dalla "casa centrale" di Broglio di Trebisacce. In: Cocchi Genick D (ed) L'età del bronzo recente in Italia, Viareggio-Lucca, pp 263-267

Grave R, Robinson E, Barbetti M, Yu Z, Bailey G, Bird R (1996-97) Analysis of South Italian pottery by PIXE-PIGME. Mediterranean Archaeology 9/10:113-125

Jones RE (2019) Chemical analysis of four decorated sherds. In: Damiani I, Parisi Presicce C (eds) La Roma dei Re. Il racconto dell'archeologia, Gangemi Editore, Roma, pp 269-270

Jones RE, Levi ST, Bettelli M, Vagnetti L (2014) Italo-Mycenaean pottery: the archaeological and Archaeometric dimensions. Incunabula Graeca 103. CNR - Istituto di Studi sul Mediterraneo Antico, Rome

Jung R, Mommsen H, Pacciarelli M (2015) From west to west: determining production regions of Mycenaean pottery of Punta di Zambrone (Calabria, Italy). JAS Reports 3:455-463

Levi ST (1999) Produzione e circulazione della ceramica nella Sibaritida protostorica I: Impasto e dolii. Prima di Sibari 1, Firenze

Levi ST, Pantano D, Sonnino M (2014) Geological environment and raw materials. In: Jones RE, Levi ST, Bettelli M, Vagnetti L (eds) op. cit, pp 111-135

Levi ST, Cannavò V, Brunelli D (2019) Atlas of ceramic fabrics 2. Archaeopress, Oxford, Southern Tyrrhenian - Pre-protohistory

Manunza MR (2016) Manufatti nuragici e micenei lungo una strada dell'età del bronzo presso Bia 'e Palma - Selargius (CA). Quaderni della Soprintendenza Archeologia, Belle Arti e Paesaggio di Cagliari 27:147-199

Maritan L (2004) Archaeometric study of Etruscan-Padan type pottery from the Veneto region: petrographic, mineralogical and geochemical-physical characterization. European J Mineralogy 17: 297-307
Peroni R (1984) Nuove ricerche sulla protostoria della Sibaritida. Roma

Peroni R (1994) Le comunità enotrie della Sibaritide ed i loro rapporti con i navigatori Egei. In: Peroni R, Trucco F (eds) Enotri e Micenei nella Sibaritide II. Altri siti della Sibaritide, Taranto, pp 832-879

Picon M (2000) Recherces préalables en vue de la détermination de l'origines des céramiques Padanes à vernis noir et à vernis rouge. In: Brogiolo P, Olcese G (eds) Produzione ceramica in area padana tra il II scolo a.C. e il VII secolo d. C: nuovi dati e prospettive di ricerca, Montava, pp 93-101

Saracino M, Maritan L, Mazzoli C (2018) Studio tecnologico della produzione ceramica dell'abitato del Bronzo finaledi Frattesina di Fratta Polesine (Rovigo) tra 'localismi e importazioni'. Bollettino del Museo Civico di Storia Naturale di Verona 42, Geologia Paleontologia Preistoria, pp 107-116

Vagnetti L (1984) Ceramica micenea e ceramica dipinta dell'età del bronzo. In: Peroni R (ed) Ricerche sulla Protostoria della Sibaritide 3, Roma, pp 164-184

Vagnetti L (2001) Le ceramiche egeo-micenee. In: Trucco F, Vagnetti L (eds) Torre Mordillo 1987-1990. Le relazioni egee di una comunità protostorica della Sibaritide. Incunabula Graeca CI, Roma, pp 299 327

Vagnetti L, Panichelli S (1994) Ceramica egea importata e di produzione locale. In: Peroni R, Trucco F (eds) Enotri e Micenei nella Sibaritide I. Broglio di Trebisacce, Taranto, pp 73-413

Vagnetti L, Bettelli M, Recchia G (2012) Catalogo delle ceramiche di tipo egeo-miceneo dall'abitato dell'età del Bronzo di Coppa Nevigata. In: Moscoloni M, Recchia G (eds) Cazzella A. Coppa Nevigata e l'area unida alla face del Candelaro durante l'età del bronzo, Foggia, pp $411-422$

Vanzetti A (2000) Broglio di Trebisacce nel quadro dell'Italia meridionale. In: Harariand M, Pearce M (eds) Il Protovillanoviano al di qua e al di là dell'Appennino. Atti della tavola rotonda (Pavia, 17 giugno 1995) Biblioteca di athenaeum 38, Como, pp 133-171

Vanzetti A et al (2014) The Broglio experiment in reproducing 'mixed Italian products. In: Jones RE, Levi ST, Bettelli M, Vagnetti L (eds.) op. Cit., pp 393-402

Williams JLL (2018) Catching a mercurial star - an appreciation. In: Bernabò Brea MA, Cultraro M, Gras M, Martinelli MC, Pouzadoux C, Spigo U (eds) Festschrift for Madeleine Cavalier. Centre Jean Bérard, Napoli, pp 59-65

Yntema D (2000) The Matt-painted Pottery of southern Italy. Lecce

Publisher's note Springer Nature remains neutral with regard to jurisdictional claims in published maps and institutional affiliations. 